Article

\title{
Optimal Design of Interval Type-2 Fuzzy Heart Rate Level Classification Systems Using the Bird Swarm Algorithm
}

\author{
Ivette Miramontes $^{1}\left(\mathbb{D}\right.$, Juan Carlos Guzman ${ }^{1} \mathbb{D}$, Patricia Melin ${ }^{1, *(\mathbb{D})}$ and \\ German Prado-Arechiga ${ }^{2}$ \\ 1 Tijuana Institute of Technology, Division of Graduate Studies and Research, 22379 Tijuana, Mexico; \\ cynthiaivette84@gmail.com (I.M.); jcguzmanpreciado89@gmail.com (J.C.J.) \\ 2 Cardio diagnostico Excel Medical Center, 22010 Tijuana, Mexico; germanprado.sinacor@gmail.com \\ * Correspondence: pmelin@tectijuana.mx
}

Received: 24 November 2018; Accepted: 12 December 2018; Published: 14 December 2018

\begin{abstract}
In this paper, the optimal designs of type- 1 and interval type-2 fuzzy systems for the classification of the heart rate level are presented. The contribution of this work is a proposed approach for achieving the optimal design of interval type-2 fuzzy systems for the classification of the heart rate in patients. The fuzzy rule base was designed based on the knowledge of experts. Optimization of the membership functions of the fuzzy systems is done in order to improve the classification rate and provide a more accurate diagnosis, and for this goal the Bird Swarm Algorithm was used. Two different type-1 fuzzy systems are designed and optimized, the first one with trapezoidal membership functions and the second with Gaussian membership functions. Once the best type-1 fuzzy systems have been obtained, these are considered as a basis for designing the interval type-2 fuzzy systems, where the footprint of uncertainty was optimized to find the optimal representation of uncertainty. After performing different tests with patients and comparing the classification rate of each fuzzy system, it is concluded that fuzzy systems with Gaussian membership functions provide a better classification than those designed with trapezoidal membership functions. Additionally, tests were performed with the Crow Search Algorithm to carry out a performance comparison, with Bird Swarm Algorithm being the one with the best results.
\end{abstract}

Keywords: heart rate; blood pressure; optimization; Bird Swarm Algorithm (BSA); type-2 fuzzy systems

\section{Introduction}

Nowadays, bio-inspired algorithms are used for optimization in different application areas, such as control [1], prediction [2], security [3], scheduling [4], etc. Optimization is defined as the mathematical process to find the best solution to a problem [5]. Several metaheuristics have been used to carry out this process, such as the Genetic Algorithm (GA) [6], Particle Swarm Optimization (PSO) [7], Flower Pollination Algorithm (FPA) [8], and Social Spider Algorithm (SSA) [9], among others. For this work, the Bird Swarm Algorithm (BSA) was used to optimize the membership function parameters of the fuzzy systems.

The Bird Swarm Algorithm [10] was originally proposed by Xian-Bing Men in 2015 to solve optimization problems. This algorithm mimics the behavior and social interaction of birds in a swarm. The behaviors imitated by this algorithm are the way in which birds search for food, flight and vigilance are performed.

BSA has been used to optimize the cost of power generation in the energy system network of both wind energy and photovoltaic solar energy [11], and variants of this algorithm have also 
been made to improve its performance, such as the Enhanced Bird Swarm Algorithm (EBSA) [12] for variable-strength combinatorial test data generation and the Chaotic Bird Swarm Optimization Algorithm (CBSA) [13], which is proposed to avoid local minimums.

With regards to fuzzy systems, these have been widely used in the medical field, specifically to provide diagnosis of diseases, such as malaria [14], leukemia [15], hepatitis [16], and meniscus tear [17], among others. In this work, a fuzzy system is presented for the classification of the heart rate level of the patients and with this, to be able to provide a medical diagnosis, in this case of the risk of having a cardiovascular event.

Initially, a type-1 fuzzy system with trapezoidal membership functions was designed to obtain the patient's heart rate classification, which is based on the experience of the cardiologist. It has as inputs the age and the trend of the heart rate, and as output has the patient's heart rate level. Also, a type-1 fuzzy system with Gaussian membership functions was designed, in the same way as the aforementioned, to find out with which type of membership functions a better classification can be provided.

To improve the classification rate and provide an even more precise diagnosis, it was proposed to realize the optimization of the membership functions for both fuzzy systems, for which different experiments are carried out using the Bird Swarm Algorithm.

Once the type-1 fuzzy systems were optimized, the problem was also addressed using interval type-2 fuzzy systems that can theoretically handle higher levels of uncertainty. The use of type-2 can consider if, in certain cases or for certain patients, the information can be very imprecise or has certain changes that could be viewed as noise or with much uncertainty.

For the design of these fuzzy systems, the initial designs of the type-1fuzzy systems are considered as the basis, and in this form an optimized and precise classification was obtained after performing different experiments. For this, the parameters of the membership functions are used, and with BSA the optimal representation of the uncertainty is search for, which means finding the optimal footprint of uncertainty.

It can also be found that in different studies, the heart rate is used as an additional input for the diagnosis of hypertension, while in this work, it is used in a separate fashion in conjunction with the nocturnal profile to determine the risk that the patient has in having a cardiovascular event.

The main contribution of this paper is the design of fuzzy systems for the classification of the heart rate level of patients. The fuzzy systems are designed based on the expert experience and tested with different architectures. Once this was done, their optimization was performed to improve the classification percentage, and for this, the Bird Swarm Algorithm was used. The reason is that when compared to other optimization methods such as the Crow Search Algorithm (CSA), the best results were obtained with BSA. Additionally, comparisons between the type- 1 and interval type- 2 fuzzy systems were performed to find out with which method a more precise classification is obtained.

This paper is organized as follows: In Section 2 the basic concepts are presented, in Section 3 the problem statement and proposed method are explained, in Section 4 the knowledge representation of the different optimized type- 1 and interval type- 2 fuzzy systems are presented, in Section 5 the results of different experiments are presented, and finally in Section 6 the conclusions and future work are presented.

\section{Basic Concepts}

\subsection{Heart Rate}

The heart rate is the number of times the heart contracts for one minute (beats per minute). As we age, changes in the rate and regularity of the heart rate may change and may signify a heart condition or other condition that must be treated by a physician. 
Heart rate can be measured in different locations of the human, including: wrists, inside of the elbow, top of the foot, and the side of the neck. To get a more accurate reading, we need to put the finger on one of the above-mentioned parts of the body and count the number of beats in $60 \mathrm{~s}$.

The resting heart rate is when the heart pumps the least amount of blood needed, which, in a healthy person, is between 60 and 100 beats per minute; however, a heart rate lower than 60 beats per minute is not necessarily a signal of a medical problem, as it may be due to some drug, or it could be a well-trained athlete, since the heart rate in athletes tends to be as low as 40 beats per minute; in Table 1 the values of heart rate based on age and sex are presented $[18,19]$.

Table 1. Heart rate values based on age and sex.

\begin{tabular}{ccccccccc}
\hline \multirow{2}{*}{ Age } & \multicolumn{9}{c}{ Men } & \multicolumn{4}{c}{ Women } \\
\cline { 2 - 8 } & Ill & Normal & Good & Excellent & Ill & Normal & Good & Excellent \\
\hline $20-29$ & 86 or more & $70-84$ & $62-68$ & 60 or less & 96 or more & $78-94$ & $72-76$ & 70 or less \\
$30-39$ & 86 or more & $72-84$ & $64-70$ & 62 or less & 98 or more & $80-96$ & $72-78$ & 70 or less \\
$40-49$ & 90 or more & $74-88$ & $66-72$ & 64 or less & 100 or more & $80-98$ & $74-78$ & 72 or less \\
50 or more & 90 or more & $76-88$ & $68-74$ & 66 o or less & 104 or more & $84-102$ & $76-86$ & 74 or less \\
\hline
\end{tabular}

Some of the factors that affect the heart rate are:

Air temperature: When temperatures and humidity soar, the heart pumps a little more blood, so the heart rate may increase, but usually no more than five to ten beats per minute.

The position of the body: At rest, sitting or standing, the heart rate is usually the same. Sometimes, when the person stands up for the first 15 to $20 \mathrm{~s}$, the heart rate may go up a bit, but after a couple of minutes, it should be set.

Emotions: If the person is stressed, anxious or "extraordinarily happy or sad" the emotions can raise the heart rate.

Body size: Body size usually does not change pulse. If the person is very obese, it is possible to see a resting heart rate greater than normal, but usually no more than 100 beats per minute.

The use of medications: Drugs that block adrenaline (beta blockers) tend to slow the heart rate, while too much thyroid medicine or too high a dose tends to raise it [18].

When the heart rate is lower than 60 beats per minute, the condition is called bradycardia, which means that the heart rate is slow or irregular, this can cause dizziness or shortness of breath in normal activities or light exercises [20]. When the heart rate is greater than 100 beats per minute it is called tachycardia, which means that the heart rate is fast or irregular. With such a high rate, the heart cannot effectively pump blood with high levels of oxygen to the body [21].

\subsection{Bird Swarm Algorithm}

The Bird Swarm Algorithm (BSA) was proposed by Xian-Bing Men in 2015 [10], which is inspired by the behavior and social interactions in swarm of birds, imitating feeding, flight and vigilance compartments to solve optimization problems.

The social behaviors of birds are simplified in 5 rules, which are described below:

Rule 1: Each bird may change between vigilance and foraging behaviors. The foraging of birds or their vigilance is modeled as a stochastic decision.

Rule 2: In foraging, each bird may record and update its best previous experience and the best previous swarm experience on the food patch. This experience may be used again to search for food. The social information is shared instantly between the entire swarm.

Rule 3: While vigilance is maintained, each bird tries to move to the center of the swarm, this behavior may be affected by the interference induced from the competition in the swarm. Birds that have higher provisions would be more likely to lie nearer to the center of the swarm than birds with low provisions of food. 
Rule 4: Birds can habitually fly to another site; when this happens, they can switch among producing and scrounging. The birds with the highest reserves of food will be producers, and those that have the lowest provisions will be scroungers. Birds with intermediate provisions would randomly choose to be a producer or scrounger.

Rule 5: Producers actively search for food. Scroungers would randomly follow a producer to search for food.

The aforementioned rules are expressed mathematically as follows:

Foraging behavior, in which each bird seeks food according to its experience and the swarms' experience, is expressed by:

$$
x_{i, j}^{t+1}=x_{i, j}^{t}+\left(p_{i, j}-x_{i, j}^{t}\right) \times C X \times \operatorname{rand}(0,1)+\left(g_{j}-x_{i, j}^{t}\right) \times S \times \operatorname{rand}(0,1),
$$

where $j$ means independent numbers uniformly distributed in $(0,1)$.

$C$ and $S$ are positive numbers, which may be called cognitive and social acceleration coefficients respectively. $P_{i, j}$ is the best previous position in the $i_{\text {th }}$ bird and $g_{j}$ is the best previous position shared in the swarm.

In vigilance behavior, each bird will try to move to the center of the swarm and compete; therefore, each bird will not move directly to the center of the swarm. This is expressed as follows:

$$
\begin{gathered}
x_{i, j}^{t+1}=x_{i, j}^{t}+A 1\left(\text { mean }_{j}-x_{i, j}^{t}\right) \times \operatorname{rand}(0,1)+A 2\left(p_{k, j}-x_{i, j}^{t}\right) \times \operatorname{rand}(-1,1) \\
A 1=a 1 \times \exp \left(-\frac{p F i t_{i}}{\text { sumFit }+\varepsilon_{1}} \times N\right) \\
A 2=a 2 \times \exp \left(\left(\frac{p F i t_{i}-p F i t_{k}}{\mid p \text { Fit }_{k}-p F i t_{i} \mid+\varepsilon}\right) \frac{N \times p F i t_{k}}{\text { sumFit }+\varepsilon}\right)
\end{gathered}
$$

where $k$ is a positive integer, chosen randomly among 1 and N. $p$ Fit $_{i}$ corresponds to the best value fitness in the ith position and sumFit signifies the sum of the best fitness value of the swarms. $\varepsilon$ is used to avoid the error in zero-division. mean $n_{j}$ is the $j$ th element of the average place of the entire swarm. $a_{1}$ and $a_{2}$ are positive constants in $(0,2)$.

Given the fact that each bird wants to stand in the center of the swarm, the product of $A 1$ and rand $(0,1)$ should not be more than $1 . A 2$ is used to simulate the direct effect induced by a specific interference when a bird moves towards the center of the swarm.

In flight behavior, birds can fly to an alternative site in response to predation threats, foraging, or for any other reason. At the new site, the birds will look again for food. The producing birds look for patches of food, while the scroungers try to feed on the food patch found by the producers. The behaviors of producers and scroungers are described as follows:

$$
\begin{gathered}
x_{i, j}^{t+1}=x_{i, j}^{t}+\operatorname{rand} n(0,1) \times x_{i, j}^{t}, \\
x_{i, j}^{t+1}=x_{i, j}^{t}+\left(x_{k, j}^{t}-x_{i, j}^{t}\right) \times F L \times \operatorname{rand}(0,1),
\end{gathered}
$$

where randn $(0,1)$ denotes a Gaussian distributed random number with mean 0 and standard deviation $1, \mathrm{k} \in[1,2,3 \ldots \mathrm{N}], \mathrm{k} \neq \mathrm{i}$. FL $(\mathrm{FL} \in[0,2])$ represents that the scrounger would follow the producer to search for food.

The authors assume, for the sake of simplicity, that each bird flies to another place every FQ unit interval, where $\mathrm{FQ}$ is a positive integer.

The BSA pseudo code is presented in Figure 1. 


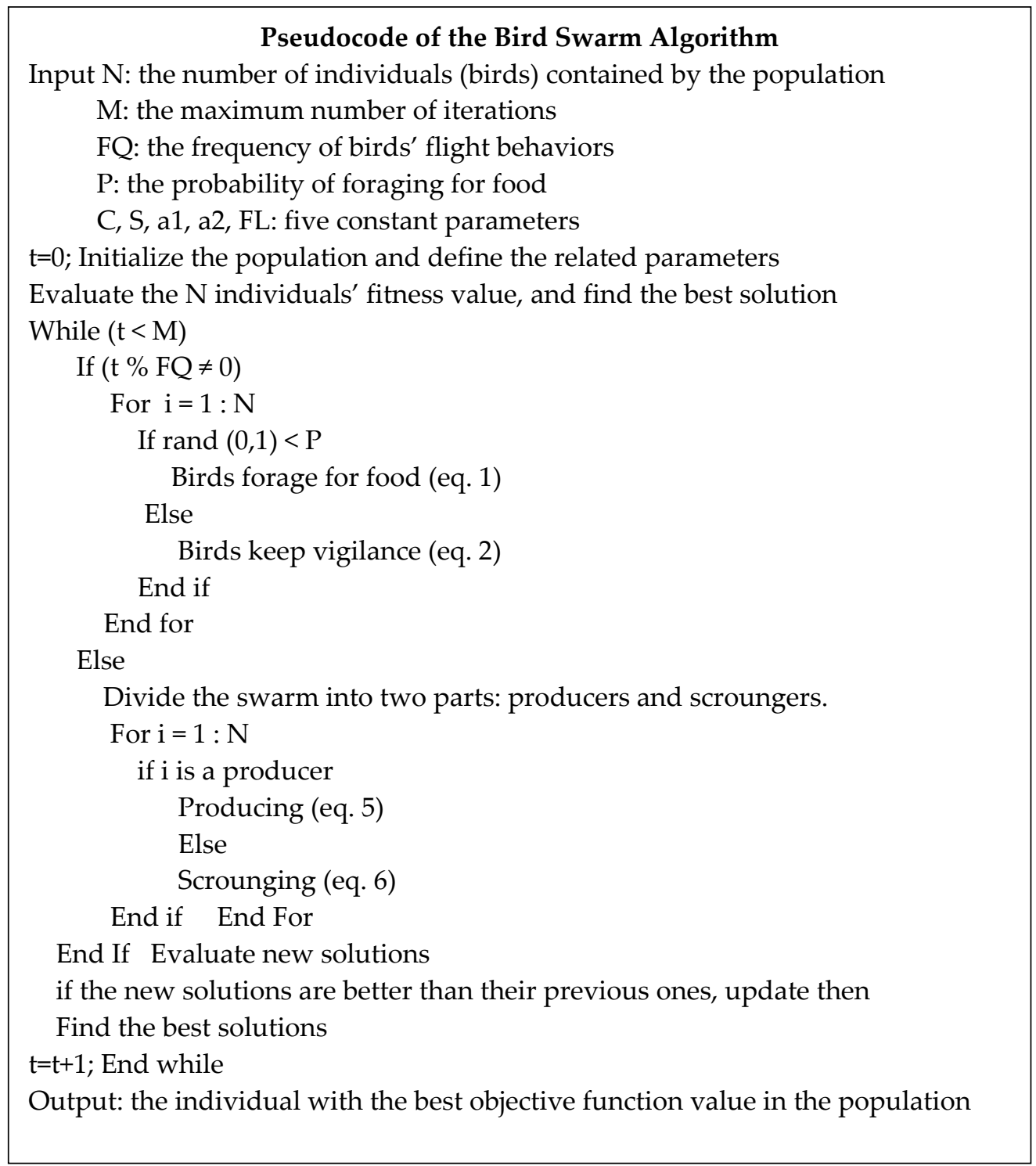

Figure 1. BSA pseudocode.

\subsection{Blood Pressure}

Blood pressure is defined as the force exerted against the walls of the arteries as the heart pumps blood through the body in order to carry the necessary oxygen and nutrients. Blood pressure is divided into two components, the first is systolic blood pressure, this refers to when the heart contracts and can be identified by the largest number at the time of examining the blood pressure. The second component is diastolic pressure, which is when the heart relaxes and is identified as the smallest number, and this is measured in millimeters of mercury ( $\mathrm{mmHg}$ ) [22-25].

The normal blood pressure in an adult is the one that goes from below $139 \mathrm{mmHg}$ in systolic pressure and below $89 \mathrm{mmHg}$ in diastolic pressure [26].

\subsection{High Blood Pressure}

Hypertension is the sustained elevation of blood pressure above normal limits, which according to the European guidelines for the management of hypertension are above $140 \mathrm{mmHg}$ in systolic pressure or above $90 \mathrm{mmHg}$ in diastolic pressure [26]. 


\subsection{Type-1 Fuzzy Logic}

Fuzzy logic was proposed by Lofti A. Zadeh [27] in 1965, and implies approximate ways of reasoning instead of exact ones, using vague, imprecise or incomplete information, which can be viewed as an attempt to construct a model of human reasoning reflecting its approximate character. Its objective is to provide a basis for approximate reasoning using imprecise propositions based on the theory of fuzzy sets.

\subsection{Interval Type-2 Fuzzy Logic}

This is an extension of type- 1 fuzzy systems proposed by Zadeh in 1975 . These were designed to mathematically represent the vagueness and uncertainty of linguistic problems. A type-2 fuzzy set is characterized by a fuzzy membership function, that is, the membership degree for each element of this set is a fuzzy set in $(0,1)$, unlike a type- 1 , where the membership grade is established as a crisp number in $(0,1)[28]$.

\subsection{Related Works}

Different works have been carried out in the medical area using type- 1 and interval type- 2 fuzzy systems (IT2FS) [28-33], which we will briefly describe next.

Sadat et al. [15] designed a type-2 fuzzy system for the diagnosis of leukemia, in which they use 14 inputs which correspond to the symptoms of leukemia and as output the diagnosis of the disease, obtaining a $94 \%$ correct classification.

For the diagnosis of hepatitis, Sotudian et al. [16] designed a type-1 and IT2FS to perform classification comparison; the fuzzy systems have ten inputs, which are attributes related to the disease, and one output, obtaining $90.0 \%$ classification with the type- 1 fuzzy system and $93.94 \%$ of classification with the interval type-2 fuzzy system.

Zarandi et al. [17] presented a type-2 fuzzy expert system for the diagnosis of meniscal tears using magnetic resonance imaging. For segmentation, first, the interval type- 2 fuzzy C-means is applied to the images, whose outputs are then used by interval type-2 possibilistic C-means to perform postprocessing. Finally, a neural network with two hidden layers is used for the classification stage. The results of the proposed type-2 expert system were compared with a well-known segmentation algorithm, confirming the superiority of the proposed system in the recognition of meniscal tears.

For dengue diagnosis, Varinder Pabbi [34] proposed a fuzzy system with 5 input variables corresponding to age, blood pressure, and other variables necessary to identify the disease, and the type of dengue that the patient has as output. The author reports having obtained good results in the diagnosis.

Mohamed and Hussein [14] designed a fuzzy system for the diagnosis and treatment of malaria, using as inputs the temperature, body mass index and pulse of the patient. The first output determines if the patient is free of malaria or, if the patient has it, the degree of complication; the second output determines the type of medication to be prescribed, obtaining as a result a high percentage of precision in the diagnosis.

\section{Problem Statement and Proposed Method}

A hybrid neural model, described in [35-37], was previously designed for the same problem, using 3 fuzzy systems: the first provides the classification of the patient's blood pressure level [38-40], the second classifies the heart rate level and a third classifies the night profile of the patient [41]. Now, in this paper, we are using the Bird Swarm Algorithm for finding the optimal design of type- 1 and type-2 fuzzy systems for the classification of the heart rate level of patients.

Because the improvement in classification is aimed at providing an accurate diagnosis to patients, it was decided to use a bio-inspired algorithm, such as the Bird Swarm Algorithm, for 
the optimization of fuzzy systems, in order for the algorithm to make the necessary adjustment in the membership functions.

Figure 2 shows the proposed methodology for what is being addressed in this work and which is explained below:

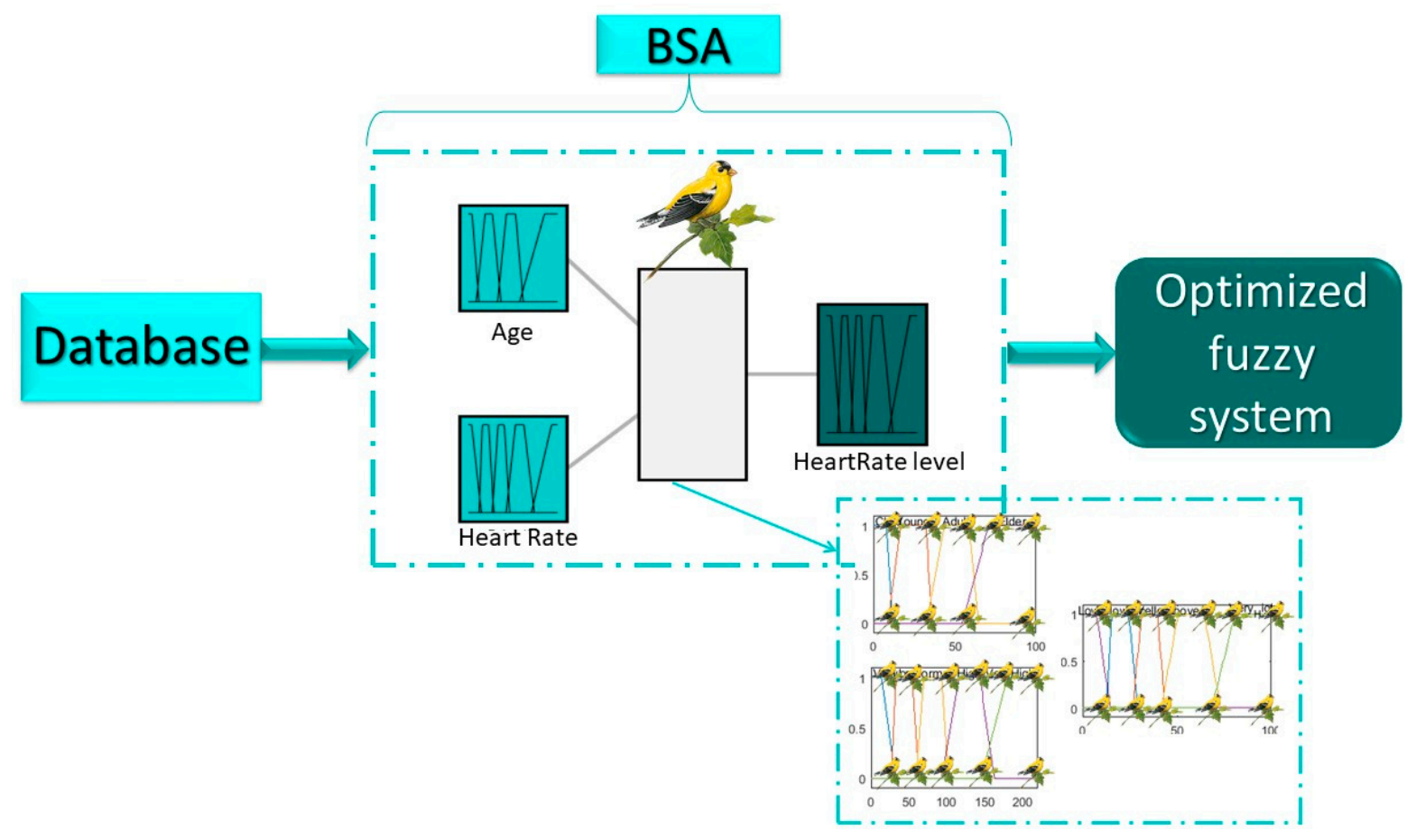

Figure 2. Proposed methodology.

A database with readings of the heart rate of a set of patients was used, which were obtained through the Ambulatory Blood Pressure Monitoring device (ABPM) [42]. To perform the optimization, 30 patients were taken from the database. The necessary information is the age and the tendency of the heart rate, and these are the inputs to the fuzzy system.

Regarding optimization, the individuals, in this case the birds, are the ones that will be moving the membership functions in the search space, until fuzzy system is found that provides a classification with less error, and for this reason, the mean square error is used as an objective function, as presented in Equation (7).

The algorithm will provide the fuzzy system with the least error in the classification, and this is tested with different sets of patients to observe its performance.

Once this is done with type-1 fuzzy systems with Gaussian and trapezoidal membership functions and the best fuzzy systems are obtained, they are taken as a basis to perform the optimization of the interval type-2 fuzzy system for which the BSA algorithm will adjust the footprint of uncertainty; for this, the procedure described above is also performed.

3.1. Design of the Type-1 Fuzzy Inference System with Trapezoidal Membership Functions for the Classification of the Heart Rate Level

A Mamdani fuzzy inference system is designed for the classification of the heart rate level based on [19] and the experience of the cardiologist, the inputs and output of which are described below and illustrated in Figure 3: 


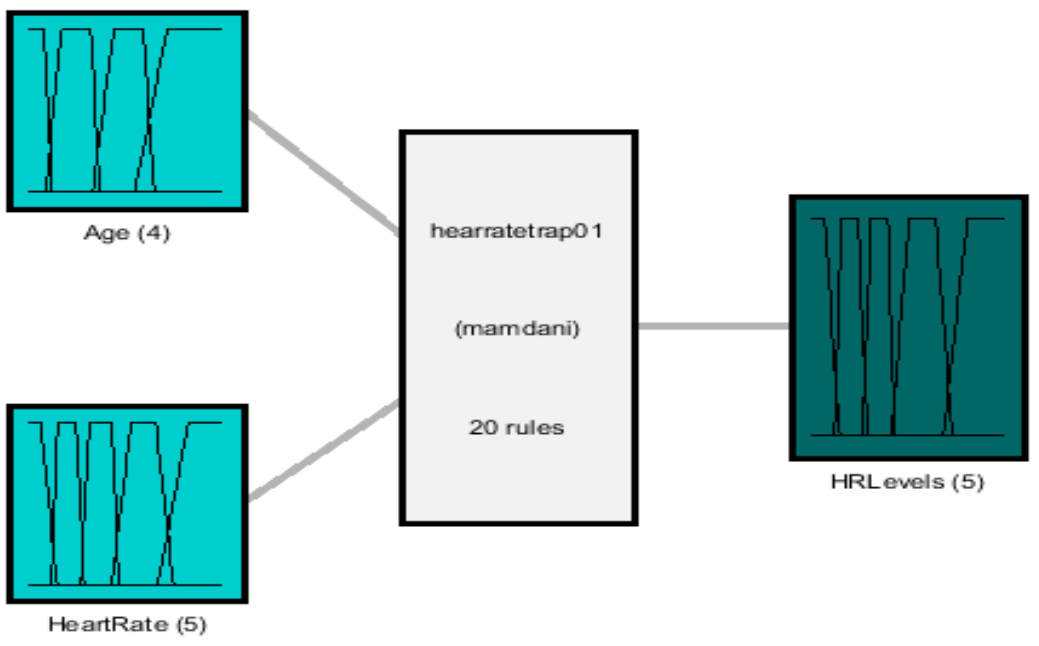

Figure 3. Heart rate level fuzzy system.

Age input: this input is in a range from 0 to 100, granulated in 4 membership functions corresponding to the linguistic variables "Child", "Young", "Adult" and "Elder", as presented in Figure 4.

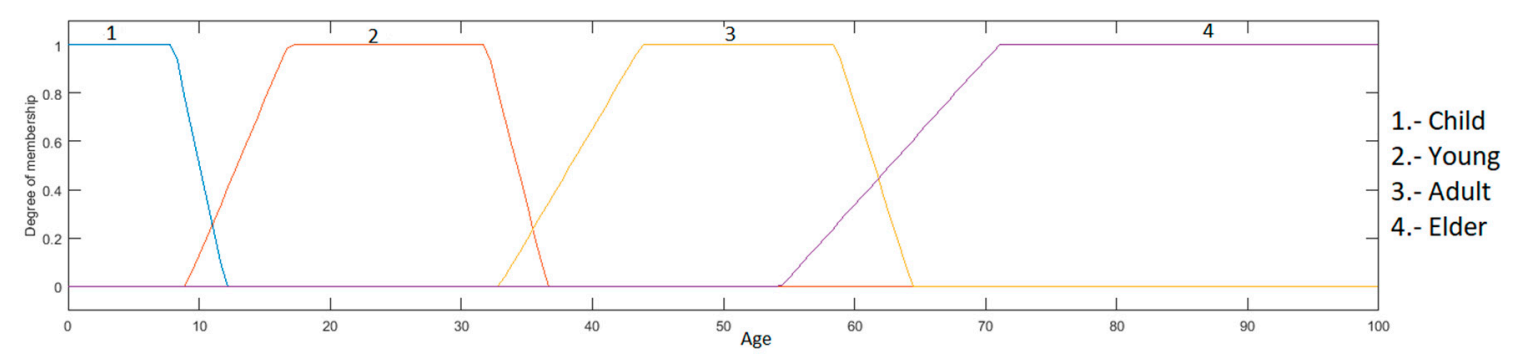

Figure 4. Input age.

Heart rate input: this input goes between the range of 0 to 220, because it is the maximum heart rate that a young person may have during physical activity, and is granulated into 5 membership functions using the linguistic variables "Very Low", "Low", "Normal", "High" and "VeryHigh", as presented in Figure 5.

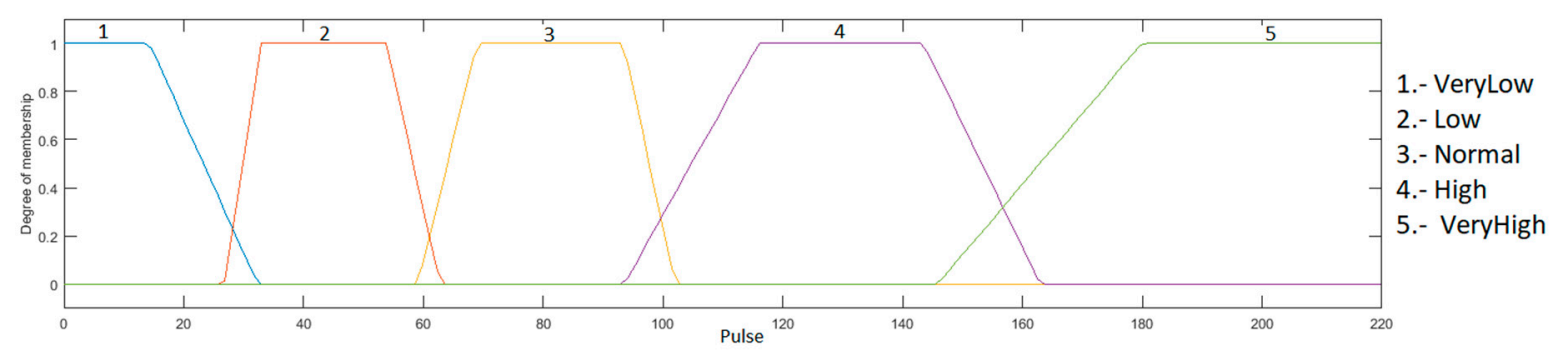

Figure 5. Input heart rate.

The output is the heart rate level, which ranges from 0 to $100 \%$; this indicates how high or low the patient's heart rate is, and is granulated into 5 membership functions, with the linguistic variables "Low", "BelowAV", "Excellent", "AboveAV" and "VeryHigh". As a defuzzification method, the centroid of area was used. The fuzzy system output is illustrated in Figure 6. 


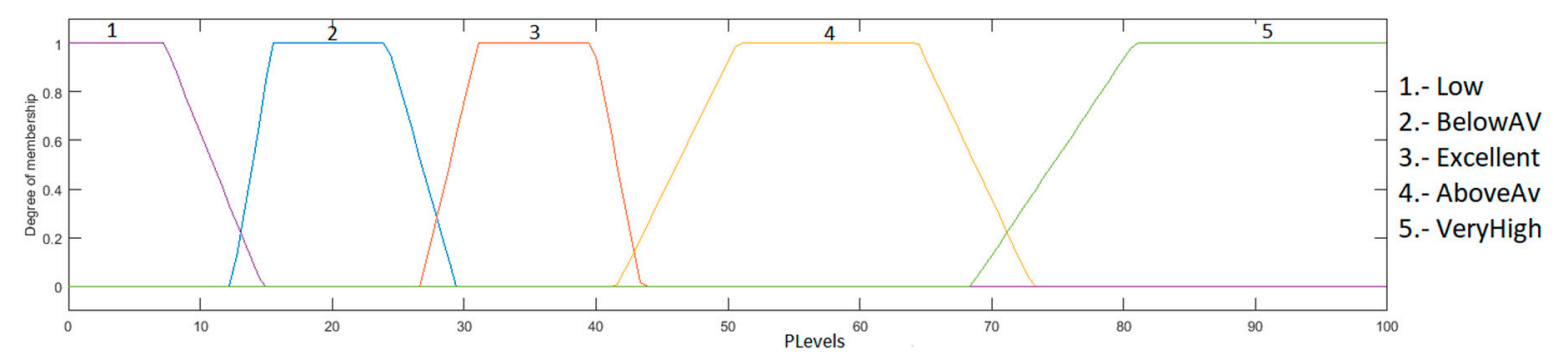

Figure 6. Output heart rate level.

3.2. Design of the Type-1 Fuzzy Inference System with Gaussian Membership Functions for the Classification of the Heart Rate Level

Age input: this is in a range of 0 to 100, granulated into 4 membership functions, having as linguistic values "Child", "Young", "Adult" and "Elder", which are presented in Figure 7.

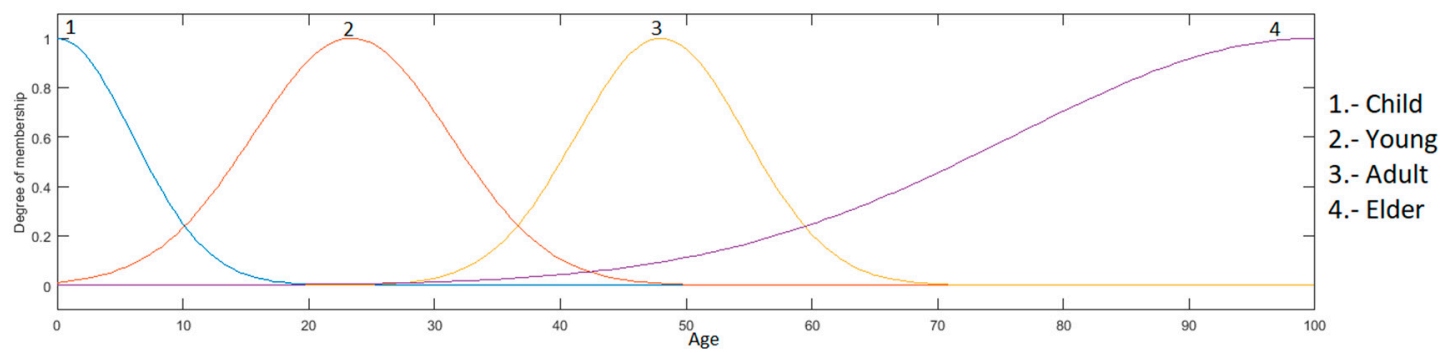

Figure 7. Input age.

Heart rate input: this input has values in the range of 0 to 220, because it is the maximum heart rate that a young person may have during physical activity, and is granulated into 5 membership functions, which correspond to the linguistics values "Very Low", "Low", "Normal", "High" and "VeryHigh". This input is presented in Figure 8.

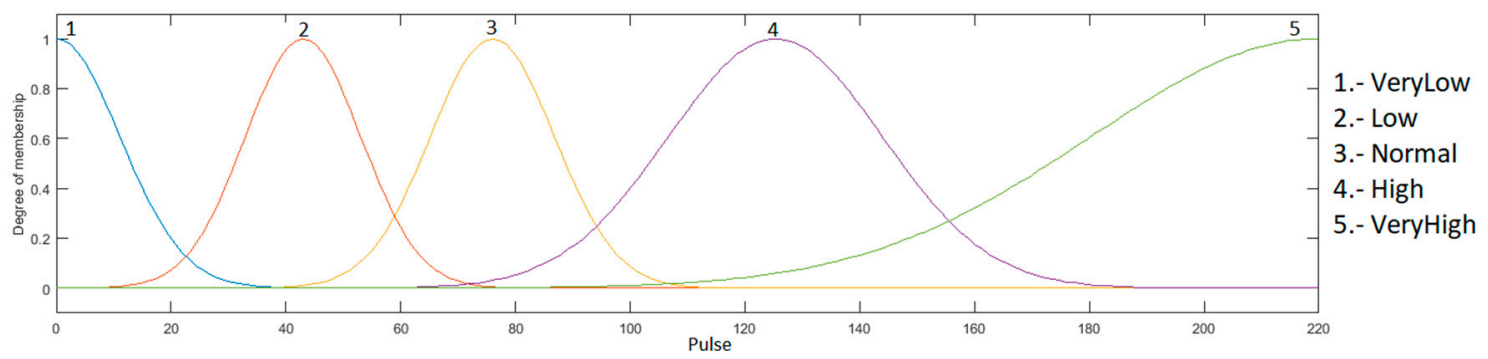

Figure 8. Input heart rate.

The output is the heart rate level, which has values in the range from 0 to $100 \%$. This indicates how high or low the heart rate of the patient is, which is granulated into 5 membership functions, using the linguistic values "Low", "BelowAV", "Excellent", "AboveAV" and "VeryHigh". As a defuzzification method, the centroid of area was used. The fuzzy system output is presented in Figure 9. 


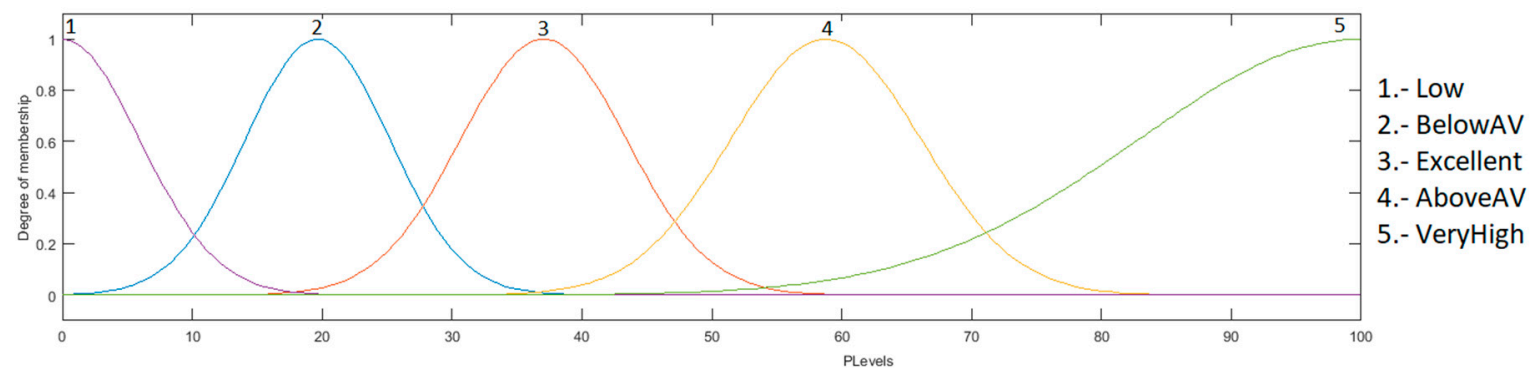

Figure 9. Output heart rate level.

The fuzzy system is formed by 20 rules, which depend on the age and heart rate to define the heart rate level of the patient, and are presented below:

1. If (Age is Child) and (Pulse is VeryLow) then (PLevels is Low) (1)

2. If (Age is Child) and (Pulse is Low) then (PLevels is Low) (1)

3. If (Age is Child) and (Pulse is Normal) then (PLevels is Excellent) (1)

4. If (Age is Child) and (Pulse is High) then (PLevels is Excellent) (1)

5. If (Age is Child) and (Pulse is VeryHigh) then (PLevels is AboveAV) (1)

6. If (Age is Young) and (Pulse is VeryLow) then (PLevels is Low) (1)

7. If (Age is Young) and (Pulse is Low) then (PLevels is BelowAV) (1)

8. If (Age is Young) and (Pulse is Normal) then (PLevels is Excellent) (1)

9. If (Age is Young) and (Pulse is High) then (PLevels is AboveAV) (1)

10. If (Age is Young) and (Pulse is VeryHigh) then (PLevels is Very_High) (1)

11. If (Age is Adult) and (Pulse is VeryLow) then (PLevels is Low) (1)

12. If (Age is Adult) and (Pulse is Low) then (PLevels is BelowAV) (1)

13. If (Age is Adult) and (Pulse is Normal) then (PLevels is Excellent) (1)

14. If (Age is Adult) and (Pulse is High) then (PLevels is AboveAV) (1)

15. If (Age is Adult) and (Pulse is VeryHigh) then (PLevels is Very_High) (1)

16. If (Age is Elder) and (Pulse is High) then (PLevels is Very_High) (1)

17. If (Age is Elder) and (Pulse is VeryHigh) then (PLevels is Very_High) (1)

18. If (Age is Elder) and (Pulse is VeryLow) then (PLevels is Low) (1)

19. If (Age is Elder) and (Pulse is Low) then (PLevels is Excellent) (1)

20. If (Age is Elder) and (Pulse is Normal) then (PLevels is Excellent) (1)

\subsection{Optimization of the Type-1 Fuzzy Inference System with the BSA}

The fuzzy systems were optimized to improve their performance using the BSA, where a search for the best parameter values for the membership functions was made with the aim of obtaining an improvement in the classification of the patients. Thirty experiments were carried out in which the parameters of the BSA were varied, as presented in Table 2. 
Table 2. Parameters used in BSA for each experiment.

\begin{tabular}{|c|c|c|c|c|c|c|c|c|c|}
\hline No & $\mathbf{M}$ & pop & DimT & DimG & FQ & c1 & c2 & a1 & a2 \\
\hline 1 & 1000 & 20 & 56 & 28 & 19 & 0.5 & 0.5 & 2 & 2 \\
\hline 2 & 870 & 24 & 56 & 28 & 28 & 0.8 & 0.8 & 1.5 & 1.5 \\
\hline 3 & 714 & 28 & 56 & 28 & 18 & 1.2 & 1.2 & 0.4 & 0.4 \\
\hline 4 & 625 & 32 & 56 & 28 & 15 & 1.5 & 1.5 & 0.1 & 0.1 \\
\hline 5 & 571 & 36 & 56 & 28 & 6 & 1.8 & 1.8 & 0.8 & 0.8 \\
\hline 6 & 574 & 38 & 56 & 28 & 21 & 2 & 2 & 1 & 1 \\
\hline 7 & 454 & 44 & 56 & 28 & 25 & 2.33 & 2.33 & 1.3 & 1.3 \\
\hline 8 & 416 & 48 & 56 & 28 & 6 & 2.48 & 2.48 & 0.6 & 0.6 \\
\hline 9 & 400 & 50 & 56 & 28 & 28 & 2.76 & 2.76 & 0.9 & 0.9 \\
\hline 10 & 357 & 56 & 56 & 28 & 20 & 3 & 3 & 1.1 & 1.1 \\
\hline 11 & 338 & 60 & 56 & 28 & 10 & 3.18 & 3.18 & 1.9 & 1.9 \\
\hline 12 & 322 & 62 & 56 & 28 & 21 & 3.22 & 3.22 & 0.5 & 0.5 \\
\hline 13 & 307 & 66 & 56 & 28 & 1 & 3.45 & 3.45 & 1.5 & 1.5 \\
\hline 14 & 285 & 70 & 56 & 28 & 13 & 3.56 & 3.56 & 0.7 & 0.7 \\
\hline 15 & 278 & 72 & 56 & 28 & 2 & 4 & 4 & 1.3 & 1.3 \\
\hline 16 & 256 & 78 & 56 & 28 & 24 & 0.4 & 0.4 & 1.8 & 1.8 \\
\hline 17 & 250 & 80 & 56 & 28 & 19 & 0.7 & 0.7 & 0.3 & 0.3 \\
\hline 18 & 235 & 86 & 56 & 28 & 1 & 1.15 & 1.15 & 0.9 & 0.9 \\
\hline 19 & 227 & 88 & 56 & 28 & 24 & 1.34 & 1.34 & 1 & 1 \\
\hline 20 & 208 & 96 & 56 & 28 & 22 & 1.45 & 1.45 & 2 & 2 \\
\hline 21 & 202 & 100 & 56 & 28 & 15 & 1.67 & 1.67 & 0.6 & 0.6 \\
\hline 22 & 166 & 120 & 56 & 28 & 4 & 1.78 & 1.78 & 0.3 & 0.3 \\
\hline 23 & 133 & 150 & 56 & 28 & 16 & 1.92 & 1.92 & 1.5 & 1.5 \\
\hline 24 & 111 & 180 & 56 & 28 & 2 & 2.18 & 2.18 & 1.2 & 1.2 \\
\hline 25 & 100 & 200 & 56 & 28 & 2 & 2.39 & 2.39 & 1.8 & 1.8 \\
\hline 26 & 95 & 210 & 56 & 28 & 21 & 2.56 & 2.56 & 0.7 & 0.7 \\
\hline 27 & 90 & 220 & 56 & 28 & 22 & 2.83 & 2.83 & 0.9 & 0.9 \\
\hline 28 & 87 & 230 & 56 & 28 & 15 & 3.4 & 3.4 & 1.5 & 1.5 \\
\hline 29 & 83 & 240 & 56 & 28 & 19 & 3.7 & 3.7 & 1.7 & 1.7 \\
\hline 30 & 80 & 250 & 56 & 28 & 20 & 4 & 4 & 2 & 2 \\
\hline
\end{tabular}

Figure 10 presents the representation of the fuzzy system with Trapezoidal membership functions, and in Figure 11, the representation of the fuzzy system with Gaussian membership functions is illustrated, with each one of the points of the membership functions adjusted by the BSA.

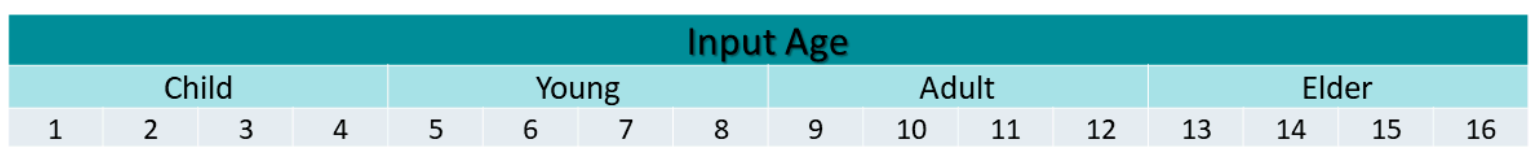

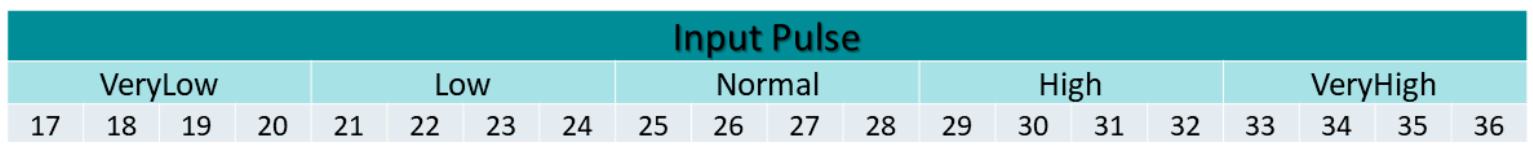

\begin{tabular}{|c|c|c|c|c|c|c|c|c|c|c|c|c|c|c|c|c|c|c|c|}
\hline \multicolumn{20}{|c|}{ Output PLevels } \\
\hline \multicolumn{4}{|c|}{ Low } & \multicolumn{4}{|c|}{ BelowAV } & \multicolumn{4}{|c|}{ Excellent } & \multicolumn{4}{|c|}{ AboveAV } & \multicolumn{4}{|c|}{ VeryHigh } \\
\hline 37 & 38 & 39 & 40 & 41 & 42 & 43 & 44 & 45 & 46 & 47 & 48 & 49 & 50 & 51 & 52 & 53 & 54 & 55 & 56 \\
\hline
\end{tabular}

Figure 10. FIS representation in BSA with trapezoidal MFs. 


\begin{tabular}{|c|c|c|c|c|c|c|c|c|c|c|c|c|c|c|c|c|}
\hline \multicolumn{8}{|c|}{ Input Age } & \multicolumn{9}{|c|}{ Input Pulse } \\
\hline & & & & & & & & Ver & Low & & & & & & gh & VeryHigh \\
\hline 1 & 2 & 3 & 4 & 5 & 6 & 7 & 8 & 9 & 10 & 11 & 12 & 13 & 14 & 15 & 16 & $17 \quad 18$ \\
\hline
\end{tabular}

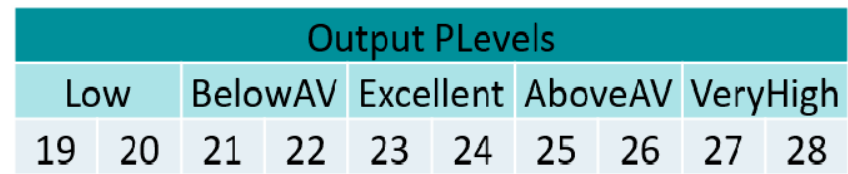

Figure 11. FIS representation in the BSA with Gaussian MFs.

As a fitness function, the mean square error is used, in order to minimize the error in classification. For this, the previous error is compared with the one obtained in the new iteration.

$$
M S E=\frac{1}{n} \sum_{i=1}^{n}\left(\hat{Y}_{i}-Y_{i}\right)^{2}
$$

Where: $\mathrm{n}=$ number of data points, $\hat{\mathrm{Y}}=$ output value by the model, $\mathrm{Y}=$ the real value for data point $\mathrm{i}$.

\subsection{Design and Optimization of the Interval Type-2 Fuzzy Systems}

Once the type-1 fuzzy systems have been optimized with the different membership functions used, they are taken as a reference for the design of the IT2FS systems to test the model. In this case, the footprint of uncertainty is symmetrical.

In Figures 12 and 13, the inputs of the fuzzy system with trapezoidal membership functions are presented, and the output of the fuzzy system with trapezoidal membership functions is presented in Figure 14.

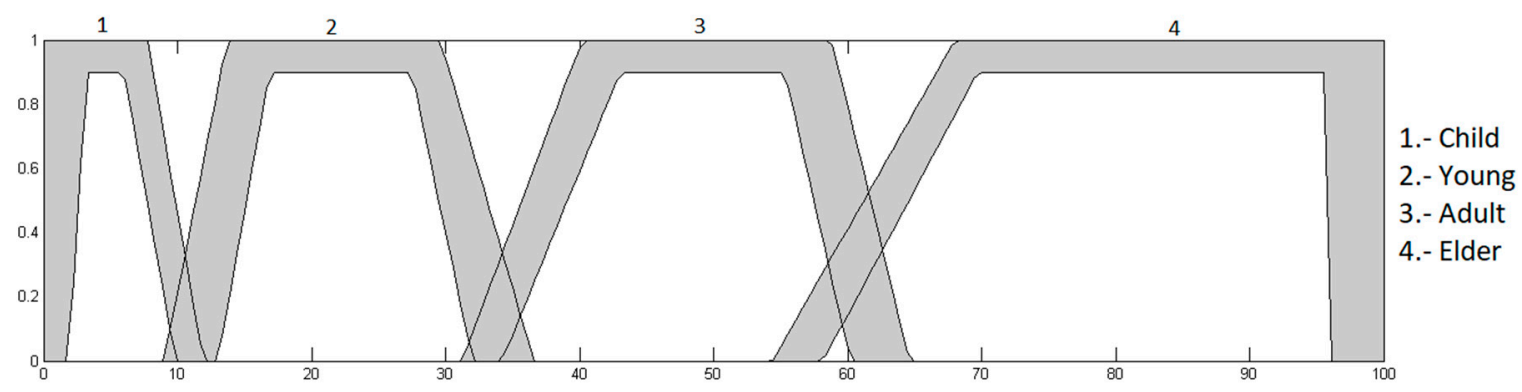

Figure 12. Age input with trapezoidal MFs.

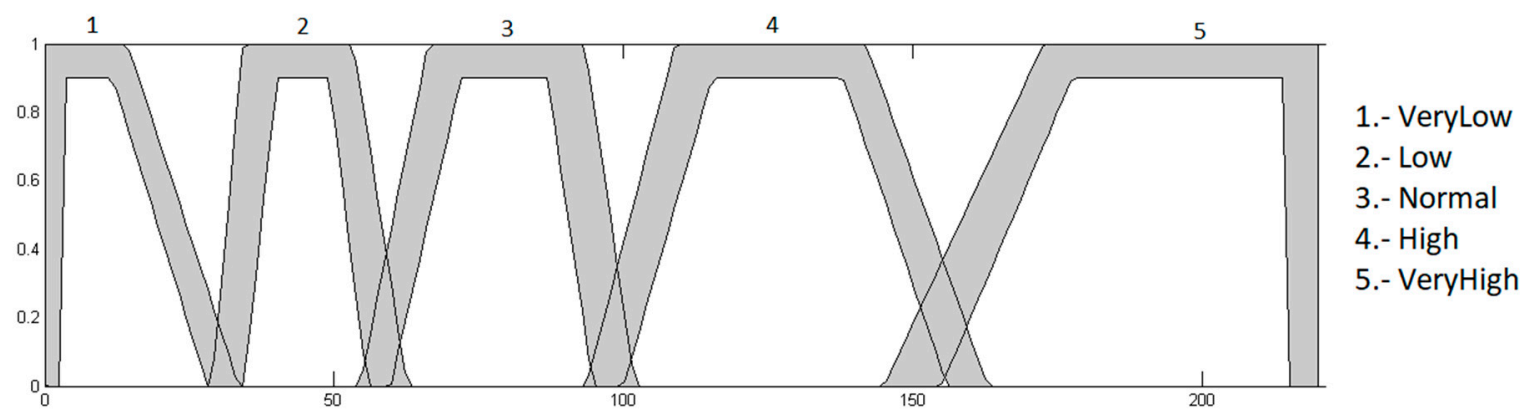

Figure 13. Input heart rate with trapezoidal MFs. 


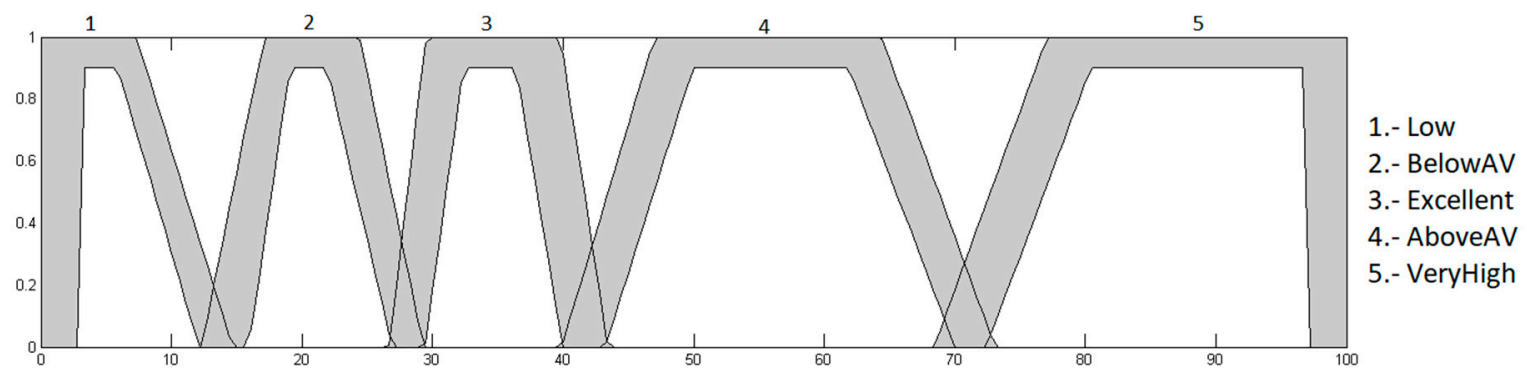

Figure 14. Output heart rate level with trapezoidal MFs.

The inputs with Gaussian membership functions are presented in Figures 15 and 16. The output of the heart rate is presented in Figure 17.

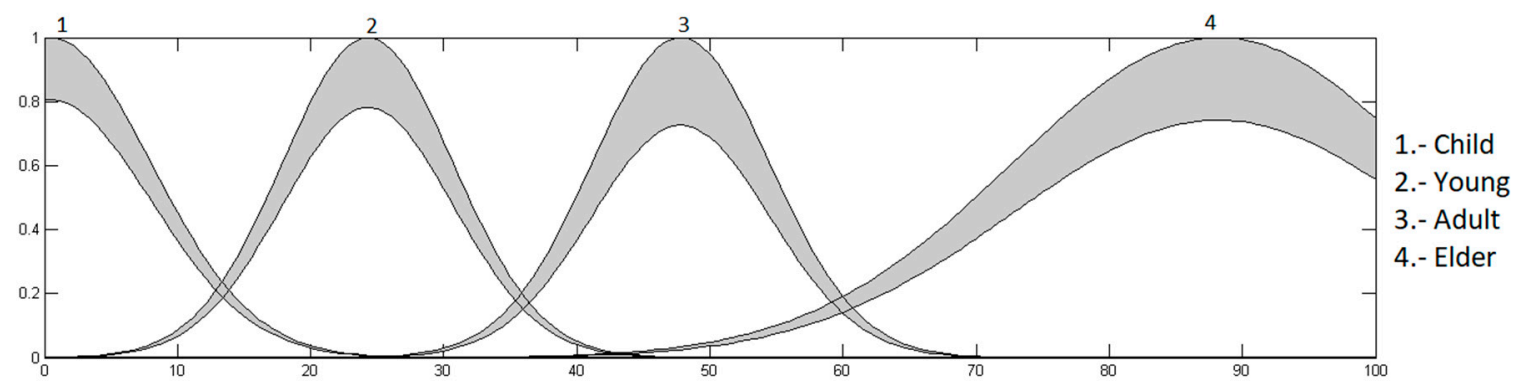

Figure 15. Age input with Gaussian MFs.

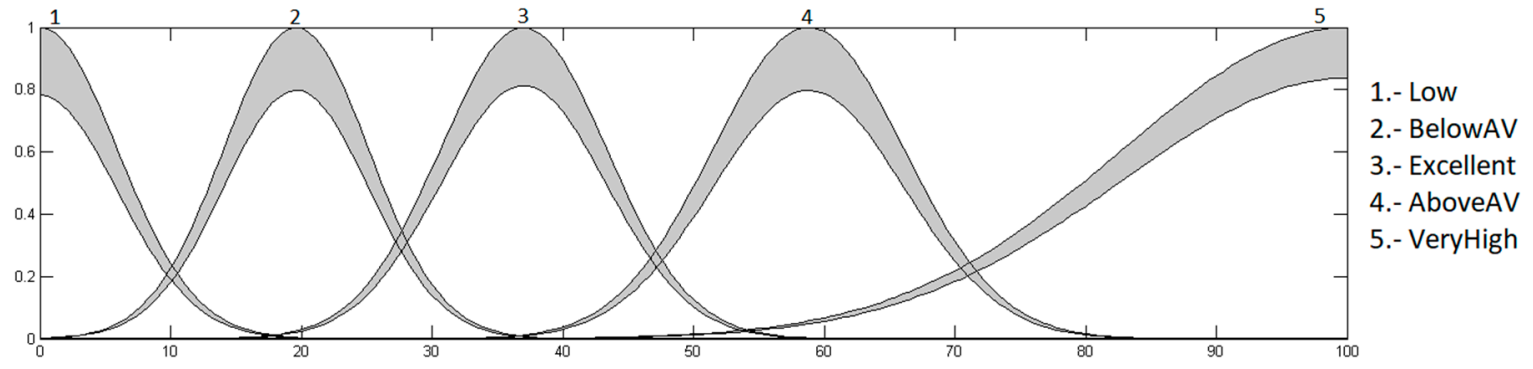

Figure 16. Input heart rate with Gaussian MFs.

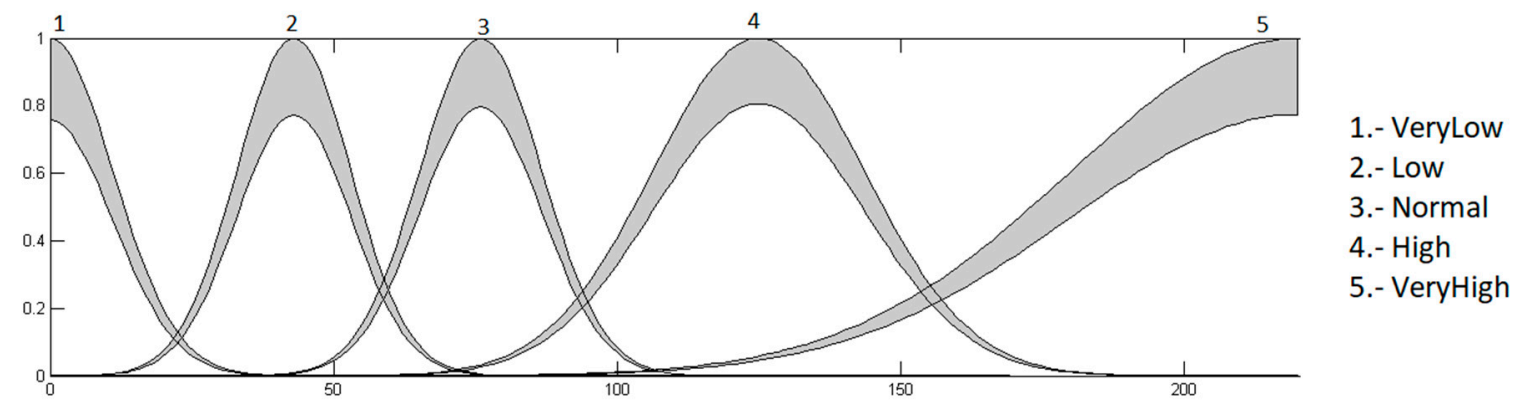

Figure 17. Output heart rate level with Gaussian MFs.

For these fuzzy systems, the footprint of uncertainty is optimized; this is to obtain its correct adjustment, and thus classification can be satisfactorily performed. Figure 18 presents the representation of the Gaussian membership functions, and for the trapezoidal membership functions, the representation of individuals is presented in Figure 10, which is the same as for the type- 1 fuzzy system. Each of these represents the points related to the footprint of uncertainty of the membership functions to be optimized. It is worth mentioning that the 20 rules above are also used in these fuzzy systems. 


\begin{tabular}{|c|c|c|c|c|c|c|c|c|}
\hline \multicolumn{4}{|c|}{ Input Age } & \multicolumn{5}{|c|}{ Input Pulse } \\
\hline Child & Young & Adult & Elder & VeryLow & Low & Normal & High & VeryHigh \\
\hline
\end{tabular}

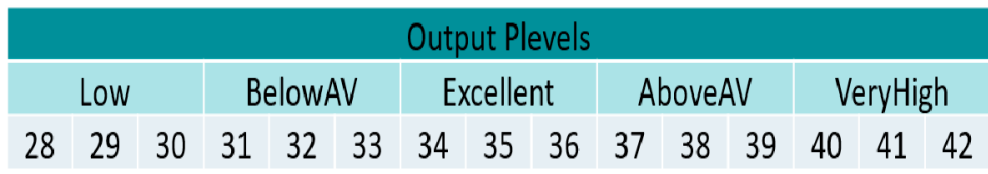

Figure 18. IT2FS representation in the BSA with Gaussians MFS.

In the same way as for the optimization of type- 1 fuzzy systems, the mean square error is used as a fitness function in order to minimize classification error, as found in Equation (7).

\section{Knowledge Representation}

4.1. Knowledge Representation of the Optimized Type-1 Fuzzy System with Trapezoidal Membership Functions

In this section, the knowledge representation of the inputs and output of the optimized type-1 fuzzy system for the classification of the pulse level with trapezoidal membership functions is presented.

\subsubsection{Input Variables}

\subsubsection{Input Age}

This input variable has the linguistic values "Child", "Young", "Adult" and "Elder". The fuzzy values for the given functions are presented as follows:

$$
\begin{aligned}
& \mu \operatorname{child}(x)=\left\{\begin{array}{c}
0, x \leq 0 \\
1,0 \leq x \leq 5.684 \\
\frac{13.76-x}{8.076}, 5.684 \leq x \leq 13.76 \\
0,13.76 \leq x
\end{array}\right. \\
& \text { Hyoung }(x)=\left\{\begin{aligned}
0, x & \leq 9 \\
\frac{x-9}{6.85}, 9 \leq x & \leq 15.85 \\
1,15.85 & \leq x \leq 31.12 \\
\frac{33.85-x}{2.73}, 31.12 & \leq x \leq 33.85 \\
0,33.85 & \leq x
\end{aligned}\right. \\
& \text { radult }(x)=\left\{\begin{aligned}
0, x \leq 32.15 & \\
\frac{x-32.15}{11.6}, 32.15 & \leq x \leq 43.75 \\
1,43.75 & \leq x \leq 55.35 \\
\frac{60.91-x}{5.56}, 55.35 & \leq x \leq 60.91 \\
0,60.91 & \leq x
\end{aligned}\right.
\end{aligned}
$$




$$
\text { pelder }(x)=\left\{\begin{array}{c}
0, x \leq 54.18 \\
\frac{x-54.18}{31.1}, 54.18 \leq x \leq 85.28 \\
1,85.28 \leq x \leq 100 \\
0,100 \leq x
\end{array}\right.
$$

\subsubsection{Input Heart Rate}

This variable has the linguistic values "Very Low", "Low", "Normal", "High" and "VeryHigh". The fuzzy values for the given functions are presented as follows:

$$
\begin{aligned}
& \text { Hverylow }(x)=\left\{\begin{array}{c}
0, x \leq 0 \\
1,0 \leq x \leq 12.38 \\
\frac{32.45-x}{19.07}, 13.38 \leq x \leq 32.45 \\
0,32.45 \leq x
\end{array}\right. \\
& \mu l o w(x)=\left\{\begin{array}{c}
0, x \leq 26.32 \\
\frac{x-26.32}{7.38}, 26.32 \leq x \leq 33.7 \\
1,33.7 \leq x \leq 48.51 \\
\frac{62.5-x}{13.99}, 48.51 \leq x \leq 62.5 \\
0,62.5 \leq x
\end{array}\right. \\
& \text { pnormal }(x)=\left\{\begin{array}{c}
0, x \leq 58.63 \\
\frac{x-58.63}{10.13}, 58.63 \leq x \leq 68.76 \\
1,68.76 \leq x \leq 85.12 \\
\frac{98.71-x}{13.59}, 85.12 \leq x \leq 98.71 \\
0,98.71 \leq x
\end{array}\right. \\
& \operatorname{phigh}(x)=\left\{\begin{array}{c}
0, x \leq 90.12 \\
\frac{x-90.12}{23.58}, 90.12 \leq x \leq 113.7 \\
1,113.7 \leq x \leq 143.2 \\
\frac{158.9-x}{15.7}, 143.2 \leq x \leq 158.9 \\
0,158.9 \leq x
\end{array}\right. \\
& \text { pveryhigh }(x)=\left\{\begin{array}{c}
0, x \leq 143.5 \\
\frac{x-143.5}{50.1}, 143.5 \leq x \leq 193.6 \\
1,193.6 \leq x \leq 220 \\
0,220 \leq x
\end{array}\right.
\end{aligned}
$$




\subsubsection{Output Variable}

This fuzzy inference system has an output variable, which corresponds to the heart rate level of the patients and has the following linguistic values: "Low", "BelowAV", "Excelent", "AboveAV" and "VeryHigh". The fuzzy values for the functions are given below:

$$
\begin{aligned}
& \mu l o w(x)=\left\{\begin{array}{c}
0, x \leq 0 \\
1,0 \leq x \leq 4.977 \\
\frac{13.4-x}{8.423}, 4.977 \leq x \leq 13.4 \\
0,13.4 \leq x
\end{array}\right. \\
& \text { Hbelowav }(x)=\left\{\begin{array}{c}
0, x \leq 10.97 \\
\frac{x-10.97}{5.77}, 10.97 \leq x \leq 16.74 \\
1,16.74 \leq x \leq 23.96 \\
\frac{27.17-x}{3.21}, 23.96 \leq x \leq 27.17 \\
0,27.17 \leq x
\end{array}\right. \\
& \text { pexcellen } t(x)=\left\{\begin{array}{c}
0, x \leq 26.55 \\
\frac{x-26.55}{4.46}, 26.55 \leq x \leq 31.01 \\
1,31.07 \leq x \leq 39.06 \\
\frac{43.84-x}{4.78}, 39.06 \leq x \leq 43.84 \\
0,43.84 \leq x
\end{array}\right. \\
& \text { paboveav }(x)=\left\{\begin{array}{c}
0, x \leq 39.42 \\
\frac{x-39.42}{8.54}, 39.42 \leq x \leq 47.96 \\
1,47.96 \leq x \leq 62.98 \\
\frac{71.81-x}{8.83}, 62.98 \leq x \leq 71.81 \\
0,71.81 \leq x
\end{array}\right. \\
& \operatorname{\mu veryhigh}(x)=\left\{\begin{array}{c}
0, x \leq 66.87 \\
\frac{x-66.87}{20.57}, 66.87 \leq x \leq 87.44 \\
1,87.44 \leq x \leq 100 \\
0,100 \leq x
\end{array}\right.
\end{aligned}
$$

4.2. Knowledge Representation of the Optimized Type-1 Fuzzy System with Gaussian Membership Functions

The knowledge representation of the optimized type-1 fuzzy system with Gaussian membership functions is presented below. 


\subsubsection{Input Variables}

\subsubsection{Input Age}

This input variable has the linguistic values "Child", "Young", "Adult" and "Elder". The fuzzy values for the given functions are presented as follows:

$$
\begin{gathered}
\operatorname{\mu child}(x)=e-\frac{1}{2}\left(\frac{x-0}{4.84}\right)^{2} \\
\mu y \text { young }(x)=e-\frac{1}{2}\left(\frac{x-24.43}{8.493}\right)^{2} \\
\operatorname{\mu adult}(x)=e-\frac{1}{2}\left(\frac{x-49.28}{6.81}\right)^{2} \\
\operatorname{\mu elder}(x)=e-\frac{1}{2}\left(\frac{x-100}{24.66}\right)^{2}
\end{gathered}
$$

\subsubsection{Input Heart Rate}

This variable has the linguistic values "Very Low", "Low", "Normal", "High" and "VeryHigh". The fuzzy values for the given functions are presented as follows:

$$
\begin{gathered}
\operatorname{verylow}(x)=e-\frac{1}{2}\left(\frac{x-0}{11.1}\right)^{2} \\
\operatorname{low}(x)=e-\frac{1}{2}\left(\frac{x-44.02}{8.602}\right)^{2} \\
\operatorname{normal}(x)=e-\frac{1}{2}\left(\frac{x-74.97}{9.143}\right)^{2} \\
\operatorname{high}(x)=e-\frac{1}{2}\left(\frac{x-125}{18.69}\right)^{2} \\
\operatorname{veryhigh}(x)=e-\frac{1}{2}\left(\frac{x-220}{38.02}\right)^{2}
\end{gathered}
$$

\subsubsection{Output Variable}

This fuzzy inference system has an output variable, which corresponds to the heart rate level of the patient's and has the following linguistic values: "Low", "BelowAV", "Excelent", "AboveAV" and "VeryHigh". The fuzzy values for the given functions are given below:

$$
\begin{aligned}
\operatorname{low}(x) & =e-\frac{1}{2}\left(\frac{x-0}{5.635}\right)^{2} \\
\operatorname{belowav}(x) & =e-\frac{1}{2}\left(\frac{x-18.56}{5.282}\right)^{2} \\
\operatorname{excellent}(x) & =e-\frac{1}{2}\left(\frac{x-37.16}{7.14}\right)^{2} \\
\operatorname{aboveav}(x) & =e-\frac{1}{2}\left(\frac{x-58.74}{7.182}\right)^{2}
\end{aligned}
$$




$$
\operatorname{verryhigh}(x)=e-\frac{1}{2}\left(\frac{x-100}{17.21}\right)^{2}
$$

4.3. Knowledge Representation of the Optimized Interval Type-2 Fuzzy System with Trapezoidal Membership Functions

The knowledge representation of the interval type-2 fuzzy system with trapezoidal membership functions is presented below.

\subsubsection{Input Variables}

\subsubsection{Input Age}

This input variable has the linguistic values "Child", "Young", "Adult" and "Elder", for each of the upper and lower membership functions. The fuzzy values for the given functions are presented as follows:

$$
\begin{aligned}
& \text { uchild }_{1}(x)=\max \left(\min \left(\frac{x-0}{0}, 1, \frac{11.53-x}{5.88}\right), 0\right) \\
& \operatorname{\mu child}_{2}(x)=\max \left(\min \left(\frac{x-3.214}{2.47}, 1, \frac{13.76-x}{6.396}\right), 0\right) \\
& \text { pyoung }_{1}(x)=\max \left(\min \left(\frac{x-9}{6.86}, 1, \frac{31.43-x}{13.09}\right), 0\right) \\
& \text { ryoung }_{2}(x)=\max \left(\min \left(\frac{x-14.02}{17.01}, 1, \frac{33.85-x}{0.65}\right), 0\right) \\
& \text { padult }_{1}(x)=\max \left(\min \left(\frac{x-32.15}{11.6}, 1, \frac{52.19-x}{4.26}\right), 0\right) \\
& \text { padult }_{2}(x)=\max \left(\min \left(\frac{x-35.58}{19.77}, 1, \frac{60.91-x}{2.97}\right), 0\right) \\
& \operatorname{\mu elder}_{1}(x)=\max \left(\min \left(\frac{x-57.01}{28.29}, 1, \frac{94.1-x}{4.6 .6}\right), 0\right) \\
& \text { pelder }_{2}(x)=\max \left(\min \left(\frac{x-61.5}{32.2}, 1, \frac{100-x}{5.7}\right), 0\right)
\end{aligned}
$$

\subsubsection{Input Heart Rate}

This variable has the linguistic values "Very Low", "Low", "Normal", "High" and "VeryHigh", for each of the upper and lower membership functions. The fuzzy values for the given functions are presented as follows:

$$
\begin{aligned}
& \operatorname{\mu verylow}_{1}(x)=\max \left(\min \left(\frac{x-0}{0}, 1, \frac{27.35-x}{21.713}\right), 0\right) \\
& \text { pverylow }_{2}(x)=\max \left(\min \left(\frac{x-5.637}{6.743}, 1, \frac{32.45-x}{14.15}\right), 0\right) \\
& \mu l o w_{1}(x)=\max \left(\min \left(\frac{x-26.32}{7.38}, 1, \frac{54.08-x}{13.69}\right), 0\right) \\
& \text { ulow }(x)=\max \left(\min \left(\frac{x-32.18}{15.7}, 1, \frac{62.5-x}{7}\right), 0\right) \\
& \text { unormal }_{1}(x)=\max \left(\min \left(\frac{x-58.63}{10.13}, 1, \frac{92.074-x}{17.13}\right), 0\right)
\end{aligned}
$$




$$
\begin{aligned}
& \text { Hnormal }_{2}(x)=\max \left(\min \left(\frac{x-66.39}{18.73}, 1, \frac{98.71-x}{7.62}\right), 0\right) \\
& \operatorname{uhigh}_{1}(x)=\max \left(\min \left(\frac{x-90.12}{23.58}, 1, \frac{148-x}{25.1}\right), 0\right) \\
& \mu h i g h_{2}(x)=\max \left(\min \left(\frac{x-95.77}{38.43}, 1, \frac{158.9-x}{24.7}\right), 0\right) \\
& \operatorname{\mu veryhigh}_{1}(x)=\max \left(\min \left(\frac{x-153.9}{28.1}, 1, \frac{210-x}{23}\right), 0\right) \\
& \text { uveryhigh }_{2}(x)=\max \left(\min \left(\frac{x-165}{33}, 1, \frac{220-x}{22}\right), 0\right)
\end{aligned}
$$

\subsubsection{Output Variable}

This fuzzy system has an output variable, which corresponds to the heart rate level of the patient's and has the following linguistic values: "Low", "BelowAV", "Excelent", "AboveAV" and "VeryHigh", for each of the lower and upper membership functions. The fuzzy values for the given functions are given below:

$$
\begin{aligned}
& \mu l o w_{1}(x)=\max \left(\min \left(\frac{x-0}{0}, 1, \frac{12-x}{9.861}\right), 0\right) \\
& \text { Hlow }(x)=\max \left(\min \left(\frac{x-1.918}{3.059}, 1, \frac{13.4-x}{6.254}\right), 0\right) \\
& \text { ubelowav } 1(x)=\max \left(\min \left(\frac{x-10.97}{5.77}, 1, \frac{22.99-x}{3.97}\right), 0\right) \\
& \text { Hbelowav } 2(x)=\max \left(\min \left(\frac{x-12.63}{11.33}, 1, \frac{27.17-x}{0.72}\right), 0\right) \\
& \text { Hexcellent }_{1}(x)=\max \left(\min \left(\frac{x-26.55}{4.46}, 1, \frac{40.47-x}{5.04}\right), 0\right) \\
& \text { Hexcellent }_{2}(x)=\max \left(\min \left(\frac{x-28.63}{10.43}, 1, \frac{43.84-x}{2.87}\right), 0\right) \\
& \text { Haboveav }_{1}(x)=\max \left(\min \left(\frac{x-41.55}{8.6}, 1, \frac{65.95-x}{5.3}\right), 0\right) \\
& \text { Haboveav }_{2}(x)=\max \left(\min \left(\frac{x-44.85}{12.4}, 1, \frac{68.65-x}{3.7}\right), 0\right) \\
& \text { pveryhigh }(x)=\max \left(\min \left(\frac{x-65.74}{21.76}, 1, \frac{95.3-x}{5.4}\right), 0\right) \\
& \text { uveryhigh }_{2}(x)=\max \left(\min \left(\frac{x-70.8}{20.7}, 1, \frac{100-x}{8.5}\right), 0\right)
\end{aligned}
$$

4.4. Knowledge Representation of the Optimized Interval Type-2 Fuzzy System with Gaussian Membership Functions

In this section, knowledge representation of the inputs and output of the optimized interval type-2 fuzzy system for the classification of the heart rate level with Gaussian membership functions is presented. 


\subsubsection{Input Variables}

\subsubsection{Input Age}

This input variable has the linguistic values "Child", "Young", "Adult" and "Elder", for each of the upper and lower membership functions. The fuzzy values for the given functions are presented as follows:

$$
\begin{aligned}
& \underline{\mu} \operatorname{child}(x)=\alpha \exp \left[-\frac{1}{2}\left(\frac{x-0}{7.72}\right)^{2}\right] \\
& \bar{\mu} \operatorname{child}(x)=\exp \left[-\frac{1}{2}\left(\frac{x-0}{7.72}\right)^{2}\right] \\
& \underline{\mu y o u n g}(x)=\alpha \exp \left[-\frac{1}{2}\left(\frac{x-23.54}{7.72}\right)^{2}\right] \\
& \bar{\mu} \text { young }(x)=\exp \left[-\frac{1}{2}\left(\frac{x-23.54}{7.72}\right)^{2}\right] \\
& \underline{\mu} \text { adult }(x)=\alpha \exp \left[-\frac{1}{2}\left(\frac{x-46.13}{5.426}\right)^{2}\right] \\
& \bar{\mu} \text { adult }(x)=\exp \left[-\frac{1}{2}\left(\frac{x-46.13}{5.426}\right)^{2}\right] \\
& \underline{\mu e l d e r}(x)=\alpha \exp \left[-\frac{1}{2}\left(\frac{x-100}{24.82}\right)^{2}\right] \\
& \bar{\mu} e l d e r(x)=\exp \left[-\frac{1}{2}\left(\frac{x-100}{24.82}\right)^{2}\right]
\end{aligned}
$$

\subsubsection{Input Heart Rate}

This variable has the linguistic values "Very Low", "Low", "Normal", "High" and "VeryHigh", for each of the upper and lower membership functions. The fuzzy values for the given functions are presented as follows:

$$
\begin{gathered}
\text { ㅅverylow }(x)=\alpha \exp \left[-\frac{1}{2}\left(\frac{x-0}{10.91}\right)^{2}\right] \\
\bar{\mu} \operatorname{verylow}(x)=\exp \left[-\frac{1}{2}\left(\frac{x-0}{10.91}\right)^{2}\right] \\
\underline{\mu l o w}(x)=\alpha \exp \left[-\frac{1}{2}\left(\frac{x-42.25}{9.739}\right)^{2}\right] \\
\bar{\mu} \operatorname{low}(x)=\exp \left[-\frac{1}{2}\left(\frac{x-42.25}{9.739}\right)^{2}\right] \\
\underline{\mu n o r m a l}(x)=\alpha \exp \left[-\frac{1}{2}\left(\frac{x-77.94}{9.381}\right)^{2}\right] \\
\bar{\mu} n \operatorname{rrmal}(x)=\exp \left[-\frac{1}{2}\left(\frac{x-77.94}{9.381}\right)^{2}\right]
\end{gathered}
$$




$$
\begin{gathered}
\underline{\mu h i g h}(x)=\alpha \exp \left[-\frac{1}{2}\left(\frac{x-127.5}{18.94}\right)^{2}\right] \\
\bar{\mu} h i g h(x)=\exp \left[-\frac{1}{2}\left(\frac{x-127.5}{18.94}\right)^{2}\right] \\
\underline{\mu v e r y h i g h}(x)=\alpha \exp \left[-\frac{1}{2}\left(\frac{x-220}{40.86}\right)^{2}\right] \\
\bar{\mu} \operatorname{veryhigh}(x)=\exp \left[-\frac{1}{2}\left(\frac{x-220}{40.86}\right)^{2}\right]
\end{gathered}
$$

\subsubsection{Output Variable}

This fuzzy system has an output variable, which corresponds to the heart rate level of the patient's and has the following linguistic values: "Low", "BelowAV", "Excelent", "AboveAV" and "VeryHigh", for each of the lower and upper membership functions. The fuzzy values for the given functions are given below:

$$
\begin{aligned}
& \underline{\mu l o w}(x)=\alpha \exp \left[-\frac{1}{2}\left(\frac{x-0}{4.432}\right)^{2}\right] \\
& \bar{\mu} l o w(x)=\exp \left[-\frac{1}{2}\left(\frac{x-0}{4.432}\right)^{2}\right] \\
& \underline{\mu b e l o w a v}(x)=\alpha \exp \left[-\frac{1}{2}\left(\frac{x-19.55}{4.236}\right)^{2}\right] \\
& \bar{\mu} \text { belowav }(x)=\exp \left[-\frac{1}{2}\left(\frac{x-19.55}{4.236}\right)^{2}\right] \\
& \text { excellent }(x)=\alpha \exp \left[-\frac{1}{2}\left(\frac{x-35.38}{4.649}\right)^{2}\right] \\
& \bar{\mu} \text { excellent }(x)=\exp \left[-\frac{1}{2}\left(\frac{x-35.38}{4.649}\right)^{2}\right] \\
& \underline{\mu} \text { aboveav }(x)=\alpha \exp \left[-\frac{1}{2}\left(\frac{x-60.25}{7.076}\right)^{2}\right] \\
& \bar{\mu} a \operatorname{bovav}(x)=\exp \left[-\frac{1}{2}\left(\frac{x-60.25}{7.076}\right)^{2}\right] \\
& \underline{\mu} \text { aboveav }(x)=\alpha \exp \left[-\frac{1}{2}\left(\frac{x-100}{17.09}\right)^{2}\right] \\
& \bar{\mu} \operatorname{abovav}(x)=\exp \left[-\frac{1}{2}\left(\frac{x-100}{17.09}\right)^{2}\right]
\end{aligned}
$$

\section{Results}

\subsection{Optimization of Type-1 Fuzzy Systems}

To carry out the optimization of the fuzzy systems, 30 experiments were performed, changing the parameters of the BSA in each case. These parameters are presented in Table 2, where $M$ is the 
number of iterations, pop the population of birds, $\operatorname{Dim} T$ is the number of dimensions used to optimize the fuzzy inference system with trapezoidal membership functions, $\operatorname{Dim} G$ the dimensions used to optimize the fuzzy system with Gaussian membership functions, $F Q(F L)$ is the frequency of bird behavior, $c 1$ and $c 2$ are the cognitive and social accelerated coefficients, respectively, and $a 1$ and $a 2$ are parameters related to the indirect and direct effects of bird vigilance behavior.

In Table 3, the parameters with the best results when performing the optimization are presented, being the same for both fuzzy inference systems.

Table 3. Parameters of the best experiment.

\begin{tabular}{cc}
\hline Parameter & Value \\
\hline M & 285 \\
pop & 70 \\
DimT & 56 \\
DimG & 28 \\
FQ & 13 \\
c1 & 3.56 \\
c2 & 3.56 \\
a1 & 0.7 \\
a2 & 0.7 \\
\hline
\end{tabular}

The percentage of classification of each experiment for both fuzzy systems are presented in Table 4, in column 2, referring to the experiments with trapezoidal membership functions. The experiments 14 and 28 were the best, with $100 \%$ classification, while column 3 refers to the experiments with Gaussian membership functions, where experiment 14 was the best, with $95 \%$ classification. The best experiments are highlighted in green.

The average classification of the 30 experiments in the fuzzy systems with trapezoidal membership functions was $94.58 \%$, while for the Gaussian membership functions it was $90.33 \%$.

In Figures 19 and 20, the inputs of the fuzzy system with optimized trapezoidal membership functions are illustrated. Likewise, in Figure 21 the output with optimized trapezoidal membership functions is presented.

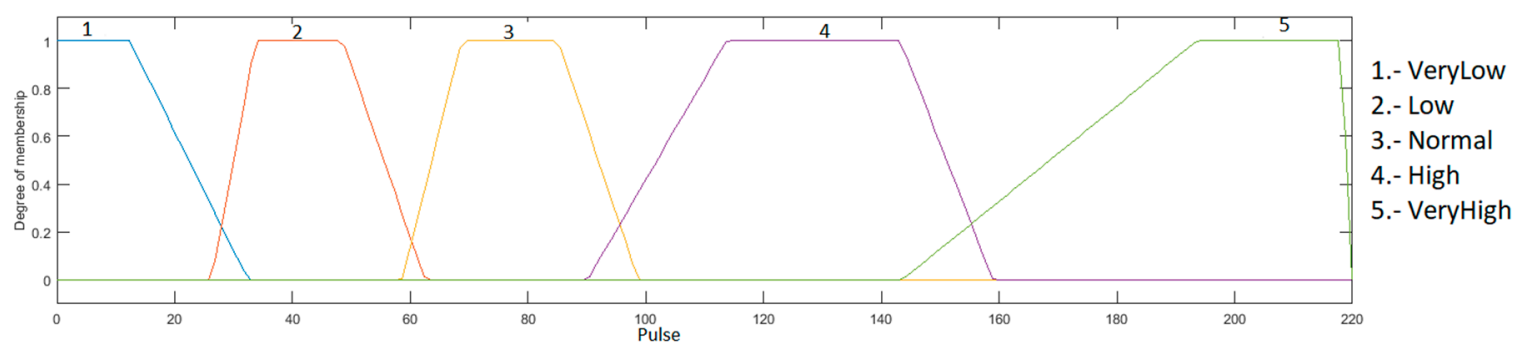

Figure 19. Optimized input age.

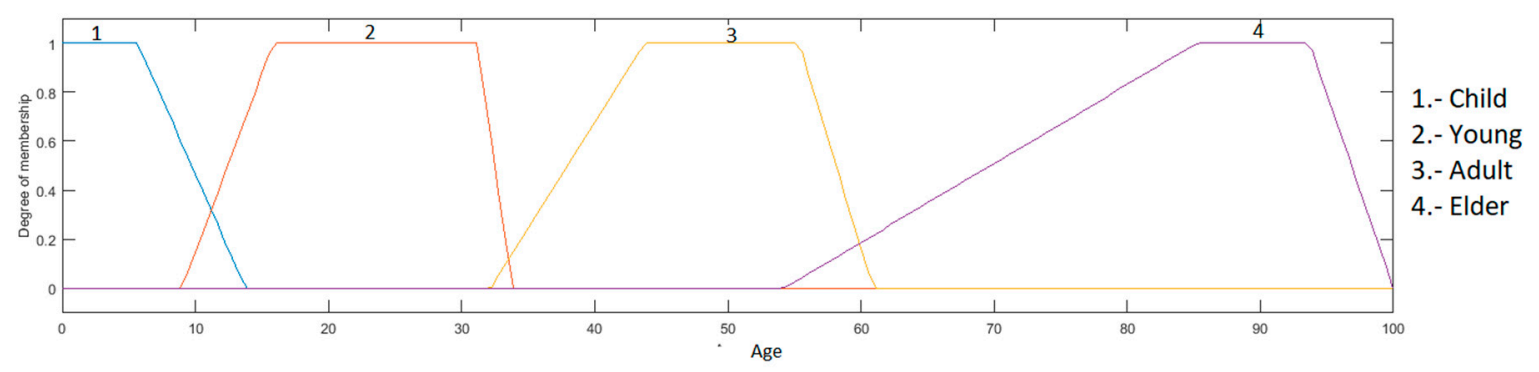

Figure 20. Optimized input heart rate. 
Table 4. Percentage of classification in each experiment with BSA.

\begin{tabular}{|c|c|c|}
\hline No. & MFTra & MFGauss \\
\hline 1 & $97.50 \%$ & $87.50 \%$ \\
\hline 2 & $95 \%$ & $87.50 \%$ \\
\hline 3 & $97.50 \%$ & $92.50 \%$ \\
\hline 4 & $90 \%$ & $87.50 \%$ \\
\hline 5 & $97.50 \%$ & $87.50 \%$ \\
\hline 6 & $90 \%$ & $92.50 \%$ \\
\hline 7 & $92.50 \%$ & $90 \%$ \\
\hline 8 & $87.50 \%$ & $92.50 \%$ \\
\hline 9 & $92.50 \%$ & $92.50 \%$ \\
\hline 10 & $95 \%$ & $90 \%$ \\
\hline 11 & $95 \%$ & $92.50 \%$ \\
\hline 12 & $90 \%$ & $95 \%$ \\
\hline 13 & $92.50 \%$ & $90 \%$ \\
\hline 14 & $100 \%$ & $95 \%$ \\
\hline 15 & $97.50 \%$ & $90 \%$ \\
\hline 16 & $95 \%$ & $90 \%$ \\
\hline 17 & $95 \%$ & $87.50 \%$ \\
\hline 18 & $97.50 \%$ & $90 \%$ \\
\hline 19 & $92.50 \%$ & $92.50 \%$ \\
\hline 20 & $95 \%$ & $92.50 \%$ \\
\hline 21 & $95 \%$ & $92.50 \%$ \\
\hline 22 & $92.50 \%$ & $85 \%$ \\
\hline 23 & $95 \%$ & $90 \%$ \\
\hline 24 & $95 \%$ & $87.50 \%$ \\
\hline 25 & $100 \%$ & $90 \%$ \\
\hline 26 & $95 \%$ & $87.50 \%$ \\
\hline 27 & $97.50 \%$ & $92.50 \%$ \\
\hline 28 & $100 \%$ & $87.50 \%$ \\
\hline 29 & $92.50 \%$ & $90 \%$ \\
\hline 30 & $90 \%$ & $92.50 \%$ \\
\hline
\end{tabular}

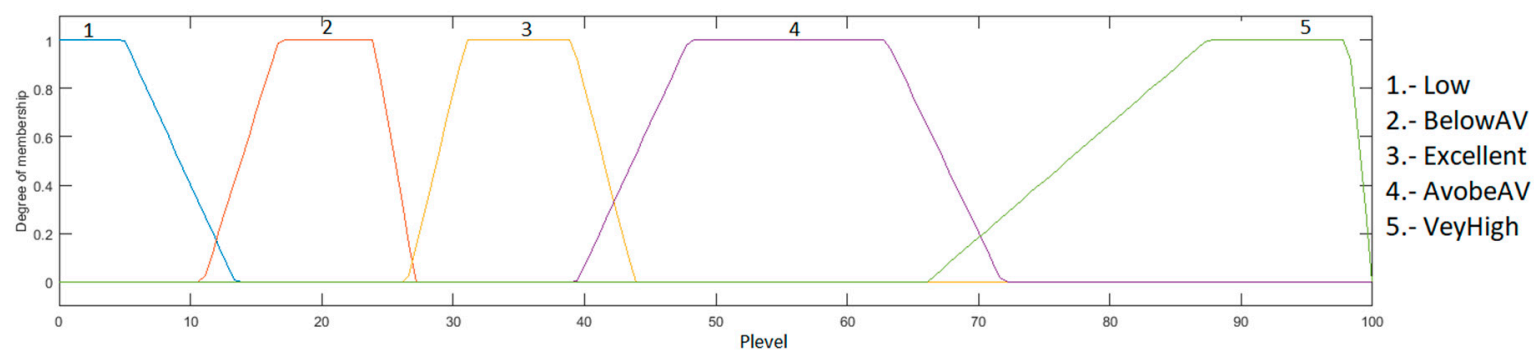

Figure 21. Optimized output heart rate level.

In the same way, in Figures 22 and 23 the inputs of the optimized fuzzy system with Gaussian membership functions are presented. In Figure 24, the output of the optimized fuzzy system with Gaussian membership function is presented. In both cases, the adjustment in each of the membership functions accomplished with the BSA can be observed. 


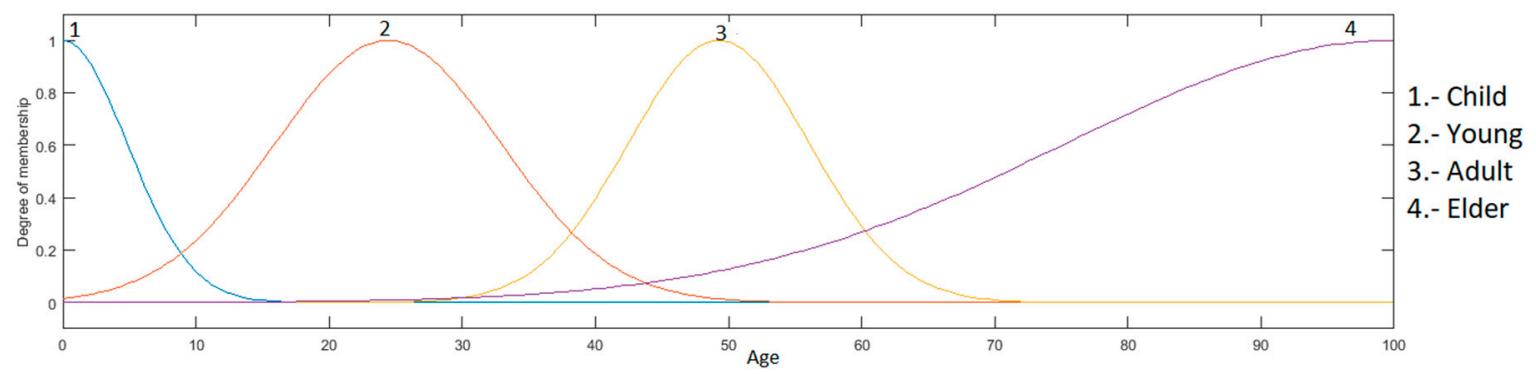

Figure 22. Optimized input age with Gaussian MFs.

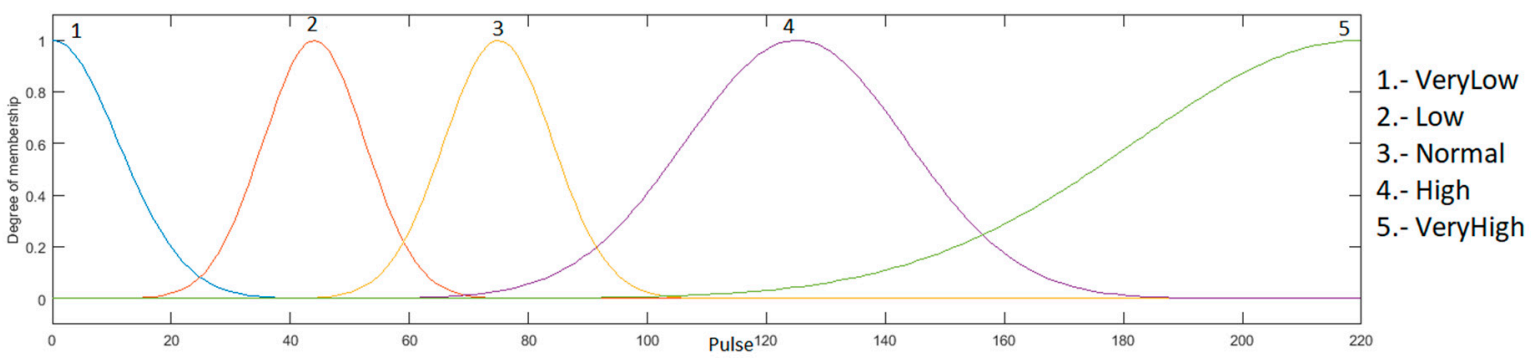

Figure 23. Optimized input heart rate with Gaussian MFs.

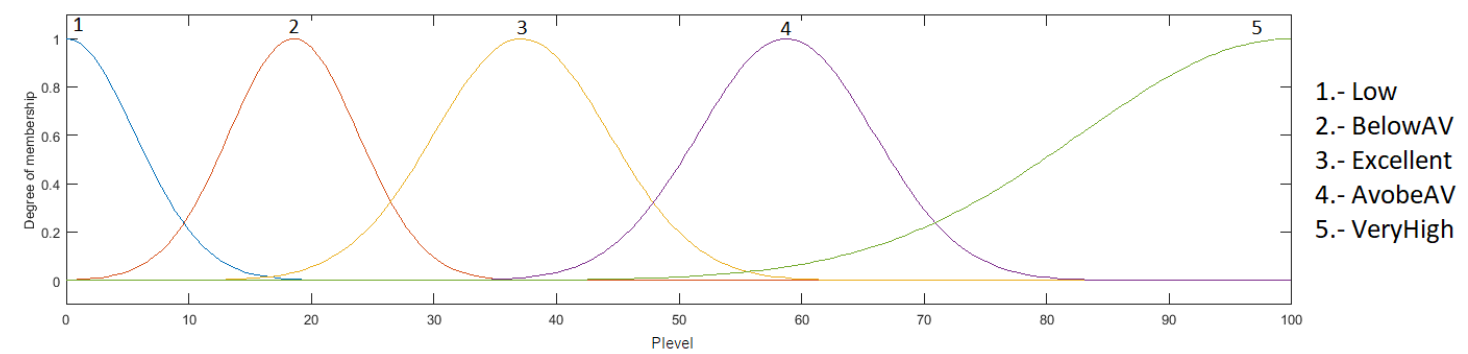

Figure 24. Optimized output heart rate level with Gaussian MFs.

Tests with the CSA were performed. In the same way as with BSA, 30 experiments were performed using the same population and iterations of Table 2, also obtaining the percentage of classification of the patients. In Table 5, the percentage of classification for each experiment is presented; column 2 presents the results with trapezoidal membership functions, while column 3 presents the results with Gaussian membership functions, in both cases presented with the type-1 fuzzy system.

The average classification of the 30 experiments in the fuzzy systems with trapezoidal membership functions was $88.33 \%$, while for the Gaussian membership functions it was $89.66 \%$.

In Table 6, the comparison of the averages of the experiments performed with CSA and BSA is presented; it can be seen that a higher percentage of classification was obtained when the optimization was done with the BSA. 
Table 5. Percentage of classification in each experiment with CSA.

\begin{tabular}{|c|c|c|}
\hline No. & MFTra & MFGauss \\
\hline 1 & $87.5 \%$ & $87.5 \%$ \\
\hline 2 & $87.5 \%$ & $92.5 \%$ \\
\hline 3 & $87.5 \%$ & $92.5 \%$ \\
\hline 4 & $85 \%$ & $87.5 \%$ \\
\hline 5 & $87.5 \%$ & $90 \%$ \\
\hline 6 & $85 \%$ & $90 \%$ \\
\hline 7 & $87.5 \%$ & $90 \%$ \\
\hline 8 & $87.5 \%$ & $90 \%$ \\
\hline 9 & $87.5 \%$ & $90 \%$ \\
\hline 10 & $85 \%$ & $90 \%$ \\
\hline 11 & $90 \%$ & $87.5 \%$ \\
\hline 12 & $90 \%$ & $90 \%$ \\
\hline 13 & $90 \%$ & $90 \%$ \\
\hline 14 & $90 \%$ & $90 \%$ \\
\hline 15 & $87.5 \%$ & $90 \%$ \\
\hline 16 & $85 \%$ & $90 \%$ \\
\hline 17 & $87.5 \%$ & $90 \%$ \\
\hline 18 & $90 \%$ & $90 \%$ \\
\hline 19 & $87.5 \%$ & $87.5 \%$ \\
\hline 20 & $90 \%$ & $87.5 \%$ \\
\hline 21 & $90 \%$ & $92.5 \%$ \\
\hline 22 & $97.5 \%$ & $90 \%$ \\
\hline 23 & $90 \%$ & $90 \%$ \\
\hline 24 & $90 \%$ & $90 \%$ \\
\hline 25 & $87.5 \%$ & $87.5 \%$ \\
\hline 26 & $87.5 \%$ & $87.5 \%$ \\
\hline 27 & $90 \%$ & $90 \%$ \\
\hline 28 & $87.5 \%$ & $90 \%$ \\
\hline 29 & $87.5 \%$ & $90 \%$ \\
\hline 30 & $87.5 \%$ & $90 \%$ \\
\hline
\end{tabular}

Table 6. Comparison of BSA and CSA averages.

\begin{tabular}{cccc}
\hline \multicolumn{2}{c}{ Trapezoidal Membership Functions } & \multicolumn{2}{c}{ Gaussians Membership Functions } \\
\hline BSA & CSA & BSA & CSA \\
$94.58 \%$ & $88.33 \%$ & $90.33 \%$ & $89.66 \%$ \\
\hline
\end{tabular}

\subsection{Optimization of the Interval Type-2 Fuzzy Systems}

For the optimization of the interval type-2 fuzzy systems, 30 different experiments were carried out, using the parameters in Table 2. Experiment 21 was the one with the best classification results for the fuzzy system with trapezoidal membership functions, with $97.5 \%$ classification. In Table 7 , the parameters of the aforementioned experiment are presented.

Table 7. Parameters of the best experiment with trapezoidal membership functions.

\begin{tabular}{cc}
\hline Parameter & Value \\
\hline M & 202 \\
pop & 100 \\
DimT & 56 \\
FQ & 15 \\
c1 & 1.67 \\
c2 & 1.67 \\
a1 & 0.6 \\
a2 & 0.6 \\
\hline
\end{tabular}


Meanwhile, for the interval type-2 fuzzy system with Gaussian membership functions, experiment 28 was the best, with a $100 \%$ classification. In Table 8, the parameters of that experiment are presented.

Table 8. Parameters of the best experiment with trapezoidal membership functions.

\begin{tabular}{cc}
\hline Parameter & Value \\
\hline M & 87 \\
pop & 230 \\
DimG & 28 \\
FQ & 15 \\
c1 & 3.4 \\
c2 & 3.4 \\
a1 & 1.5 \\
a2 & 1.5 \\
\hline
\end{tabular}

Table 9 presents the percentage of classification in each experiment for both membership functions, having a classification average for interval type-2 fuzzy systems with trapezoidal membership functions of $92.92 \%$, while for the interval type-2 fuzzy system with Gaussian membership functions an average rating of $92.78 \%$. The best experiments are highlight in green.

Table 9. Percentage of classification in each experiment with BSA.

\begin{tabular}{ccc}
\hline No & MFTra & MFGauss \\
\hline 1 & $92.50 \%$ & $92.50 \%$ \\
2 & $92.50 \%$ & $90 \%$ \\
3 & $92.50 \%$ & $87.50 \%$ \\
4 & $95 \%$ & $90 \%$ \\
5 & $92.50 \%$ & $87.50 \%$ \\
6 & $92.50 \%$ & $92.50 \%$ \\
7 & $92.50 \%$ & $95 \%$ \\
8 & $92.50 \%$ & $90 \%$ \\
9 & $92.50 \%$ & $92.50 \%$ \\
10 & $92.50 \%$ & $95 \%$ \\
11 & $92.50 \%$ & $97.50 \%$ \\
12 & $92.50 \%$ & $87.50 \%$ \\
13 & $92.50 \%$ & $95 \%$ \\
14 & $92.50 \%$ & $92.50 \%$ \\
15 & $95 \%$ & $92.50 \%$ \\
16 & $92.50 \%$ & $85 \%$ \\
17 & $95 \%$ & $92.50 \%$ \\
18 & $92.50 \%$ & $92.50 \%$ \\
19 & $92.50 \%$ & $92.50 \%$ \\
20 & $95 \%$ & $97.50 \%$ \\
21 & $97.50 \%$ & $90 \%$ \\
22 & $87.50 \%$ & $97.50 \%$ \\
23 & $92.50 \%$ & $100 \%$ \\
24 & $92.50 \%$ & $92.50 \%$ \\
25 & $92.50 \%$ & $87.50 \%$ \\
26 & $92.50 \%$ & $90 \%$ \\
27 & $92.50 \%$ & $97.50 \%$ \\
28 & $92.50 \%$ & $100 \%$ \\
29 & $92.50 \%$ & $97.50 \%$ \\
30 & $95 \%$ & $92.50 \%$ \\
\hline & & \\
\hline & & \\
\hline & & \\
\hline
\end{tabular}

The optimized inputs with trapezoidal membership function are presented in Figures 25 and 26, while the output is presented in Figure 27. 


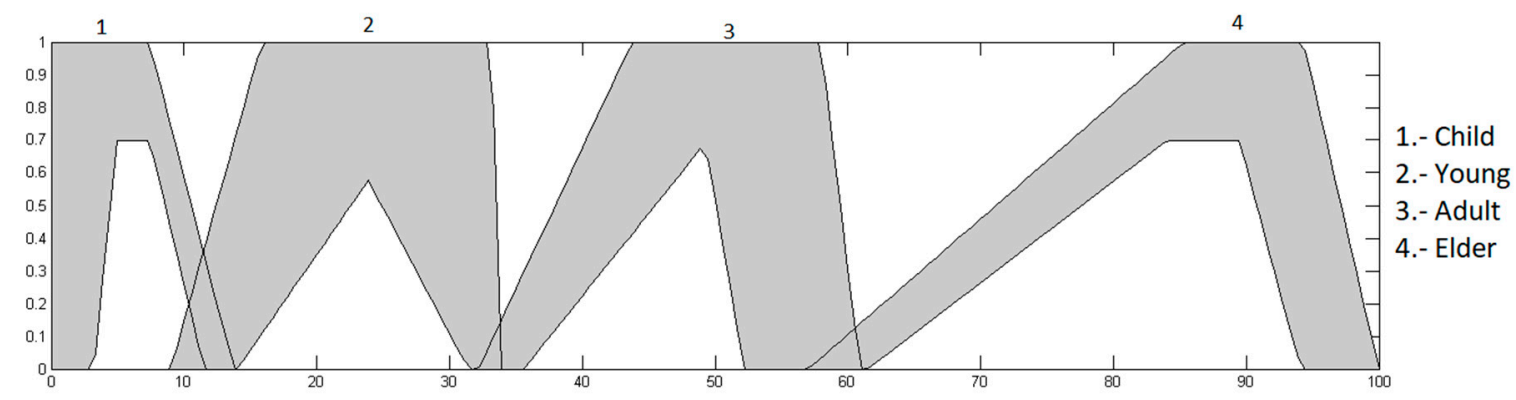

Figure 25. Optimized input age with trapezoidal MFs.

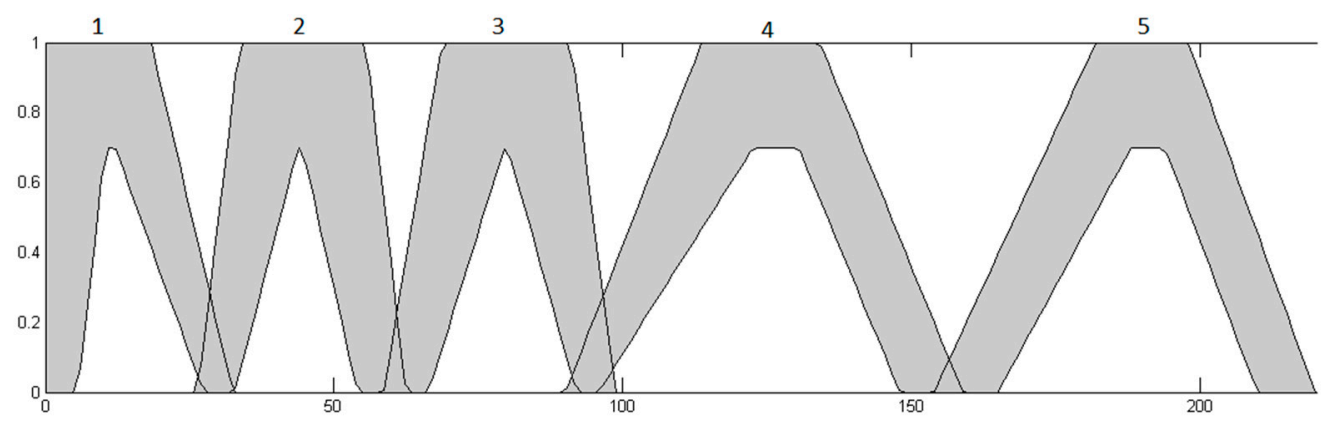

1.- VeryLow

2.- Low

3.- Normal

4.- High

5.- VeryHigh

Figure 26. Optimized input heart rate with trapezoidal MFs.

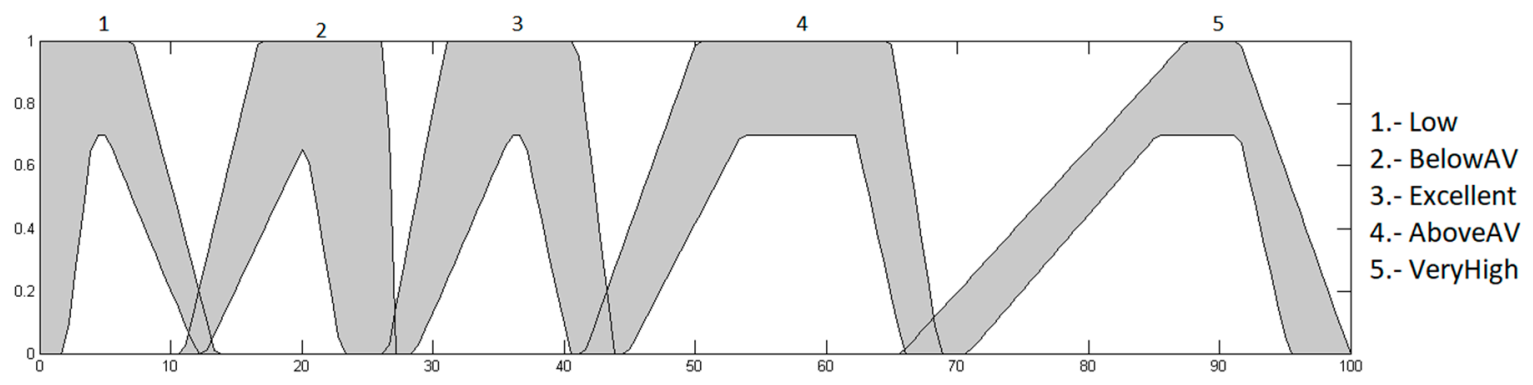

Figure 27. Optimized output heart rate level with trapezoidal MFs.

Similarly, in Figures 28 and 29, the inputs of optimized interval type-2 fuzzy system with Gaussian membership functions are presented. The output of this fuzzy system is presented in Figure 30.

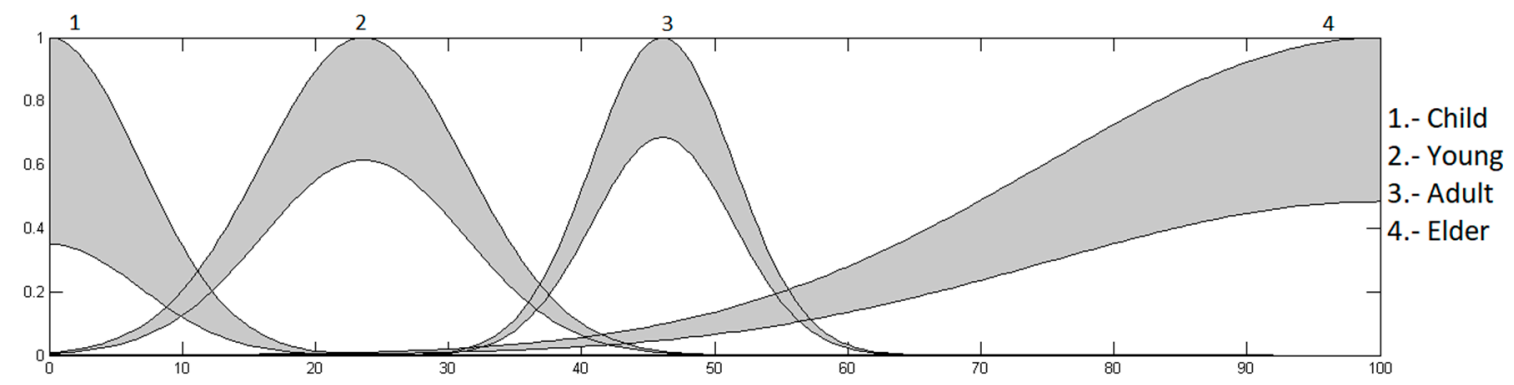

Figure 28. Optimized input age with Gaussian MFs. 


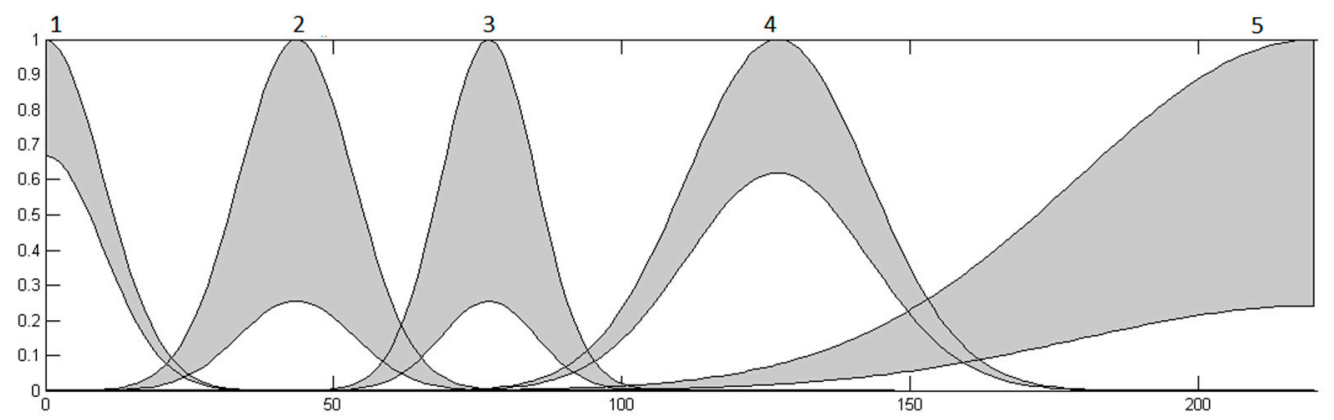

1.- VeryLow

2.- Low

3.- Normal

4.- High

5.- VeryHigh

Figure 29. Optimized input heart rate with Gaussian MFs.

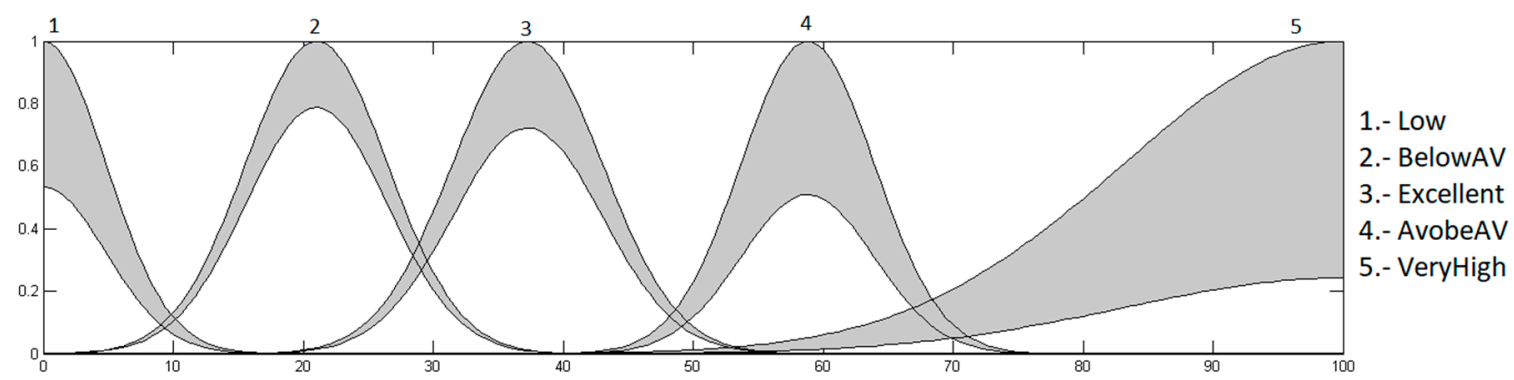

Figure 30. Optimized output heart rate level with Gaussian MFs.

In this case, the adjustment in the footprint of uncertainty is more noticeable than the optimizations of the type-1 fuzzy systems.

30 experiments with the CSA were performed, using the same population and iterations presented in Table 2, and also obtaining the percentage of classification of the patients. In Table 10, the percentage of classification for each experiment is presented; column 2 presents the results with trapezoidal membership functions, while column 3 presents the results with Gaussian membership functions in both cases being presented with the interval type- 2 fuzzy system.

The average classification of the 30 experiments in the fuzzy systems with trapezoidal membership functions was $92.83 \%$, while for the Gaussian membership functions it was $88.33 \%$.

In Table 11, the comparison of the averages of the experiments performed with CSA and BSA is presented, it can be seen that a higher percentage of classification was obtained when the optimization was done with the BSA. 
Table 10. Percentage of classification in each experiment with CSA.

\begin{tabular}{|c|c|c|}
\hline No & MFTra & MFGauss \\
\hline 1 & $92.50 \%$ & $92.50 \%$ \\
\hline 2 & $90 \%$ & $92.50 \%$ \\
\hline 3 & $90 \%$ & $92.50 \%$ \\
\hline 4 & $90 \%$ & $95 \%$ \\
\hline 5 & $90 \%$ & $92.50 \%$ \\
\hline 6 & $90 \%$ & $95 \%$ \\
\hline 7 & $90 \%$ & $92.50 \%$ \\
\hline 8 & $90 \%$ & $92.50 \%$ \\
\hline 9 & $90 \%$ & $92.50 \%$ \\
\hline 10 & $90 \%$ & $87.50 \%$ \\
\hline 11 & $90 \%$ & $95 \%$ \\
\hline 12 & $90 \%$ & $92.50 \%$ \\
\hline 13 & $90 \%$ & $95 \%$ \\
\hline 14 & $90 \%$ & $92.50 \%$ \\
\hline 15 & $90 \%$ & $95 \%$ \\
\hline 16 & $90 \%$ & $92.50 \%$ \\
\hline 17 & $90 \%$ & $95 \%$ \\
\hline 18 & $90 \%$ & $95 \%$ \\
\hline 19 & $90 \%$ & $92.50 \%$ \\
\hline 20 & $90 \%$ & $95 \%$ \\
\hline 21 & $90 \%$ & $95 \%$ \\
\hline 22 & $90 \%$ & $95 \%$ \\
\hline 23 & $90 \%$ & $92.50 \%$ \\
\hline 24 & $90 \%$ & $92.50 \%$ \\
\hline 25 & $90 \%$ & $87.50 \%$ \\
\hline 26 & $90 \%$ & $92.50 \%$ \\
\hline 27 & $90 \%$ & $92.50 \%$ \\
\hline 28 & $90 \%$ & $92.50 \%$ \\
\hline 29 & $90 \%$ & $87.50 \%$ \\
\hline 30 & $90 \%$ & $92.50 \%$ \\
\hline
\end{tabular}

Table 11. Comparison of BSA and CSA averages.

\begin{tabular}{cccc}
\hline \multicolumn{2}{c}{ Tapezoidal Membership Functions } & \multicolumn{2}{c}{ Gaussians Membership Functions } \\
\hline BSA & CSA & BSA & CSA \\
$92.92 \%$ & $92.83 \%$ & $92.78 \%$ & $88.33 \%$ \\
\hline
\end{tabular}

\subsection{Tests with Patients Using the Type-1 Fuzzy Systems}

Tests were carried out with 15 random patients; this is because in our group of patients, there is not much variation in age and, usually, the level of the pulse is found to be in the excellent range, and we wanted to observe that the fuzzy system made classifications correctly for different ages and heart rate levels.

Table 12 presents the age and pulse of the randomized patients when BelowAV is the value below average, Excnt is excellent, $A b o v e A V$ is above average and Vhigh is very high value. In this case, the non-optimized fuzzy system with trapezoidal membership functions classified 13 patients correctly, and the optimized fuzzy system classified all 15 patients correctly. On the other hand, the non-optimized Gaussian fuzzy system classified 12 patients correctly, and the optimized fuzzy system classified 14 patients correctly. The patients classified incorrectly are highlighted in red. 
Table 12. Test with random patients.

\begin{tabular}{|c|c|c|c|c|c|c|c|}
\hline \multirow[b]{2}{*}{ No. } & \multirow[b]{2}{*}{ Age } & \multirow[b]{2}{*}{ Pulse } & \multirow[b]{2}{*}{ Real } & \multicolumn{2}{|c|}{ Trapezoidal } & \multicolumn{2}{|c|}{ Gaussian } \\
\hline & & & & $\begin{array}{c}\text { No Optimized } \\
\text { FIS }\end{array}$ & $\begin{array}{l}\text { Optimized } \\
\text { FIS }\end{array}$ & $\begin{array}{c}\text { No Optimized } \\
\text { FIS }\end{array}$ & $\begin{array}{l}\text { Optimized } \\
\text { FIS }\end{array}$ \\
\hline 1 & 25 & 84 & Excnt & Excnt & Excnt & Excnt & Excnt \\
\hline 2 & 83 & 95 & AboveAV & Excnt & AboveAV & AboveAV & AboveAV \\
\hline 3 & 15 & 114 & AboveAV & AboveAV & AboveAV & AboveAV & AboveAV \\
\hline 4 & 34 & 72 & Excnt & Excnt & Excnt & Excnt & Excnt \\
\hline 5 & 42 & 135 & AboveAV & AboveAV & AboveAV & AboveAV & AboveAV \\
\hline 6 & 91 & 97 & VHigh & AboveAV & VHigh & AboveAV & VHigh \\
\hline 7 & 45 & 60 & BelowAV & BelowAV & BelowAV & Excnt & Excnt \\
\hline 8 & 56 & 87 & Excnt & Excnt & Excnt & AboveAV & Exc \\
\hline 9 & 75 & 102 & VHigh & VHigh & VHigh & VHigh & VHigh \\
\hline 10 & 9 & 120 & Excnt & Excnt & Excnt & Excnt & Excnt \\
\hline 11 & 14 & 92 & Excnt & Excnt & Excnt & Excnt & Excnt \\
\hline 12 & 38 & 78 & Excnt & Excnt & Excnt & Excnt & Excnt \\
\hline 13 & 29 & 80 & Excnt & Excnt & Excnt & Excnt & Excnt \\
\hline 14 & 21 & 62 & Excnt & Excnt & Excnt & Excnt & Excnt \\
\hline 15 & 6 & 115 & Excnt & Excnt & Excnt & Excnt & Excnt \\
\hline
\end{tabular}

Table 13 presents the results of the classification of 20 patients from our database, with the non-optimized fuzzy system with trapezoidal membership functions correctly classifying 19 patients, as well as the optimized fuzzy system. In the case of the non-optimized fuzzy system with Gaussian membership functions, 19 patients were classified correctly, while the optimized fuzzy system did not have a single error in the classification.

Table 13. Test with real patients.

\begin{tabular}{|c|c|c|c|c|c|c|c|}
\hline \multirow[b]{2}{*}{ No. } & \multirow[b]{2}{*}{ Age } & \multirow[b]{2}{*}{ Pulse } & \multirow[b]{2}{*}{ Real } & \multicolumn{2}{|c|}{ Trapezoidal } & \multicolumn{2}{|c|}{ Gaussian } \\
\hline & & & & $\begin{array}{l}\text { No Optimized } \\
\text { FIS }\end{array}$ & $\begin{array}{l}\text { Optimized } \\
\text { FIS }\end{array}$ & $\begin{array}{l}\text { No Optimized } \\
\text { FIS }\end{array}$ & $\begin{array}{l}\text { Optimized } \\
\text { FIS }\end{array}$ \\
\hline 1 & 46 & 75 & Excnt & Excnt & Excnt & Excnt & Excnt \\
\hline 2 & 28 & 88 & Excnt & Excnt & Excnt & Excnt & Excnt \\
\hline 3 & 30 & 69 & Excnt & Excnt & Excnt & Excnt & Excnt \\
\hline 4 & 33 & 59 & BelowAV & BelowAV & BelowAV & Excnt & BelowAV \\
\hline 5 & 31 & 68 & Excnt & Excnt & Excnt & Excnt & Excnt \\
\hline 6 & 32 & 71 & Excnt & Excnt & Excnt & Excnt & Excnt \\
\hline 7 & 32 & 66 & Excnt & Excnt & Excnt & Excnt & Excnt \\
\hline 8 & 27 & 66 & Excnt & Excnt & Excnt & Excnt & Excnt \\
\hline 9 & 31 & 72 & Excnt & Excnt & Excnt & Excnt & Excnt \\
\hline 10 & 30 & 76 & Excnt & Excnt & Excnt & Excnt & Excnt \\
\hline 11 & 32 & 81 & Excnt & Excnt & Excnt & Excnt & Excnt \\
\hline 12 & 28 & 76 & Excnt & Excnt & Excnt & Excnt & Excnt \\
\hline 13 & 31 & 85 & Excnt & Excnt & Excnt & Excnt & Excnt \\
\hline 14 & 26 & 85 & Excnt & Excnt & Excnt & Excnt & Excnt \\
\hline 15 & 31 & 77 & Excnt & Excnt & Excnt & Excnt & Excnt \\
\hline 16 & 29 & 77 & Excnt & Excnt & Excnt & Excnt & Excnt \\
\hline 17 & 45 & 69 & Excnt & Excnt & Excnt & Excnt & Excnt \\
\hline 18 & 27 & 63 & Excnt & Excnt & Excnt & Excnt & Excnt \\
\hline 19 & 25 & 107 & AvobeAV & AvobeAV & AvobeAV & AvobeAV & AvobeAV \\
\hline 20 & 25 & 95 & AvobeAV & Excnt & Excnt & Excnt & AvobeAV \\
\hline
\end{tabular}

\subsection{Tests with Patients Using Interval Type-2 Fuzzy Systems}

In the same way as with the type-1 fuzzy systems, interval type-2 (optimized and non-optimized) fuzzy systems were tested to compare their results, considering the classification results of 15 random patients in Table 14. In this case, the fuzzy system with non-optimized trapezoidal membership 
functions classified 13 patients correctly, as did the optimized fuzzy system. The fuzzy system with non-optimized Gaussian membership functions classified 12 patients correctly, while the optimized one classified all 15 patients correctly.

Table 14. Test with random patients using the IT2FS.

\begin{tabular}{cccccccc}
\hline & & & & \multicolumn{2}{c}{ Trapezoidal } & \multicolumn{2}{c}{ Gaussian } \\
\cline { 5 - 7 } No. & Age & Pulse & Real & $\begin{array}{c}\text { No Optimized } \\
\text { FIS }\end{array}$ & $\begin{array}{c}\text { Optimized } \\
\text { FIS }\end{array}$ & $\begin{array}{c}\text { No Optimized } \\
\text { FIS }\end{array}$ & $\begin{array}{c}\text { Optimized } \\
\text { FIS }\end{array}$ \\
\hline 1 & 25 & 84 & Excnt & Excnt & Excnt & Excnt & Excnt \\
2 & 83 & 95 & AvobeAV & AvobeAV & AvobeAV & AvobeAV & AvobeAV \\
3 & 15 & 114 & AvobeAV & AvobeAV & AvobeAV & AvobeAV & AvobeAV \\
4 & 34 & 72 & V & Exc & Exc & Exc & Exc \\
5 & 42 & 135 & AvobeAV & AvobeAV & AvobeAV & AvobeAV & AvobeAV \\
6 & 91 & 97 & VHigh & AboveAV & VHigh & AboveAV & VHigh \\
7 & 45 & 60 & BelowAV & Excnt & Excnt & Excnt & BelowAV \\
8 & 56 & 87 & Excnt & Excnt & Excnt & AboveAV & Excnt \\
9 & 75 & 102 & VHigh & VHigh & VHigh & VHigh & VHigh \\
10 & 9 & 120 & Excnt & Excnt & Excnt & Excnt & Excnt \\
11 & 14 & 92 & Excnt & Excnt & AboveAV & Excnt & Excnt \\
12 & 38 & 78 & Excnt & Excnt & Excnt & Excnt & Excnt \\
13 & 29 & 80 & Excnt & Excnt & Excnt & Excnt & Excnt \\
14 & 21 & 62 & Excnt & Excnt & Excnt & Excnt & Excnt \\
15 & 6 & 115 & Excnt & Excnt & Excnt & Excnt & Excnt \\
\hline
\end{tabular}

Table 15 presents classification results with interval type-2 fuzzy systems with our patients. As can be seen, the non-optimized fuzzy system with trapezoidal membership functions classified 19 patients correctly, the non-optimized fuzzy system with Gaussian membership functions classified 18 patients correctly, while optimized fuzzy systems classified all patients correctly.

Table 15. Test with real patients.

\begin{tabular}{|c|c|c|c|c|c|c|c|}
\hline \multirow[b]{2}{*}{ No. } & \multirow[b]{2}{*}{ Age } & \multirow[b]{2}{*}{ Pulse } & \multirow[b]{2}{*}{ Real } & \multicolumn{2}{|c|}{ Trapezoidal } & \multicolumn{2}{|c|}{ Gaussian } \\
\hline & & & & $\begin{array}{l}\text { No Optimized } \\
\text { FIS }\end{array}$ & $\begin{array}{l}\text { Optimized } \\
\text { FIS }\end{array}$ & $\begin{array}{l}\text { No Optimized } \\
\text { FIS }\end{array}$ & $\begin{array}{l}\text { Optimized } \\
\text { FIS }\end{array}$ \\
\hline 1 & 46 & 75 & Excnt & Excnt & Excnt & Excnt & Excnt \\
\hline 2 & 28 & 88 & Excnt & Excnt & Excnt & Excnt & Excnt \\
\hline 3 & 30 & 69 & Excnt & Excnt & Excnt & Excnt & Excnt \\
\hline 4 & 33 & 59 & BelowAV & Excnt & BelowAV & Excnt & BelowAV \\
\hline 5 & 31 & 68 & Excnt & Excnt & Excnt & Excnt & Excnt \\
\hline 6 & 32 & 71 & Excnt & Excnt & Excnt & Excnt & Excnt \\
\hline 7 & 32 & 66 & Excnt & Excnt & Excnt & Excnt & Excnt \\
\hline 8 & 27 & 66 & Excnt & Excnt & Excnt & Excnt & Excnt \\
\hline 9 & 31 & 72 & Excnt & Excnt & Excnt & Excnt & Excnt \\
\hline 10 & 30 & 76 & Excnt & Excnt & Excnt & Excnt & Excnt \\
\hline 11 & 32 & 81 & Excnt & Excnt & Excnt & Excnt & Excnt \\
\hline 12 & 28 & 76 & Excnt & Excnt & Excnt & Excnt & Excnt \\
\hline 13 & 31 & 85 & Excnt & Excnt & Excnt & Excnt & Excnt \\
\hline 14 & 26 & 85 & Excnt & Excnt & Excnt & Excnt & Excnt \\
\hline 15 & 31 & 77 & Excnt & Excnt & Excnt & Excnt & Excnt \\
\hline 16 & 29 & 77 & Excnt & Excnt & Excnt & Excnt & Excnt \\
\hline 17 & 45 & 69 & Excnt & Excnt & Excnt & Excnt & Excnt \\
\hline 18 & 27 & 63 & Excnt & Excnt & Excnt & Excnt & Excnt \\
\hline 19 & 25 & 107 & AboveAV & AboveAV & AboveAV & AboveAV & AboveAV \\
\hline 20 & 25 & 95 & AboveAV & AboveAV & AboveAV & Excnt & AboveAV \\
\hline
\end{tabular}


Table 16 summarizes the percentages of classification of the fuzzy inference systems for the 15 random patients.

Table 16. Classification with type-1 and interval type-2 fuzzy systems with random patients.

\begin{tabular}{cccccccc}
\hline \multicolumn{2}{c}{ FST1 No Optimized } & \multicolumn{2}{c}{ FST1 Optimized } & \multicolumn{2}{c}{ FST2 No Optimized } & \multicolumn{2}{c}{ FST2 Optimized } \\
\hline Trap & Gauss & Trap & Gauss & Trap & Gauss & Trap & Gauss \\
$86.6 \%$ & $80 \%$ & $100 \%$ & $93.3 \%$ & $86.6 \%$ & $80 \%$ & $86.6 \%$ & $100 \%$ \\
\hline
\end{tabular}

Table 17 presents the percentages of classification in the fuzzy inference systems for the 20 real patients.

Table 17. Classification with type- 1 and interval type-2 fuzzy systems with real patients.

\begin{tabular}{cccccccc}
\hline \multicolumn{2}{c}{ FST1 No Optimized } & \multicolumn{2}{c}{ FST1 Optimized } & \multicolumn{2}{c}{ FST2 No Optimized } & \multicolumn{2}{c}{ FST2 Optimized } \\
\hline Trap & Gauss & Trap & Gauss & Trap & Gauss & Trap & Gauss \\
$95 \%$ & $90 \%$ & $95 \%$ & $100 \%$ & $95 \%$ & $90 \%$ & $100 \%$ & $100 \%$ \\
\hline
\end{tabular}

It can be observed in Tables 16 and 17 that the fuzzy systems, both type- 1 and interval type- 2 with Gaussian membership functions, achieved a $100 \%$ classification in most of the experiments performed with the different sets of patients, and in which the improvement of classification when optimized was also remarkable, given that the lowest value for the non-optimized fuzzy system was $80 \%$ correct classification.

\section{Discussion}

Optimization has been applied in a large number of areas using different bio-inspired algorithms to find the best solution to different types of problems. In industry, we can find how genetic algorithms are implemented to maximize the useful life of a high-performance controller in the drilling process, as well as the material removal rate [43]. With regard to the optimization of artificial neural networks, the harmony search algorithm has been used to train the echo estate neural networks [44], which are a special form of recurrent neural networks.

Optimization of fuzzy controllers has been carried out applying the gray wolf optimizer, to find the best parameters of a Takagi-Sugeno proportional-integral fuzzy controllers for a class of non-linear servo systems [45], and in another case was used to optimize a Takagi-Sugeno fuzzy PI controller for the flux and conductivity control of Reverse Osmosis Desalination Plants [46].

In this work, BSA was used for the optimization of the membership function parameters in type-1 and interval type-2 fuzzy systems, with the aim of improving the performance at the moment of classifying the heart level of different patients. The optimization was performed in the same way with the CSA; in Table 6 the comparison of the results is presented when the type- 1 fuzzy systems are optimized, showing that for both membership functions, using the BSA provides a better result. Meanwhile, in Table 11, the results are presented when optimizing the footprint of uncertainty of interval type-2 fuzzy systems. In the case when the trapezoidal membership functions were optimized, the improvement is minimal when the BSA algorithm is compared with the CSA; on the contrary, for optimization of fuzzy system with Gaussian membership functions, it is observed that the BSA was better.

Different tests were performed with patients in order to decide which of the optimized fuzzy systems yields a better classification, and the results were compared with non-optimized fuzzy systems, as presented in Tables 16 and 17, noting that, for the different sets of patients, better results were obtained with the fuzzy systems optimized with Gaussian membership functions, since they classified $100 \%$ of patients correctly in both cases. 


\section{Conclusions}

In this work, the design, implementation, and optimization of fuzzy systems for the classification of the heart level is presented. The algorithm chosen for this optimization was the BSA, with which a total of 30 different experiments were carried out with type- 1 and IT2FS, varying the parameters of the algorithm until good results were obtained. The experiments described above were made in the same way as with the CSA, and when performing the comparison of results, a better classification was obtained when optimizing with the BSA.

Experiments were performed with different sets of patients, testing the fuzzy systems, which produced a better percentage of classification when optimized, as well as the non-optimized fuzzy systems to compare the classification provided.

Once this was done and the results were compared, it could be observed that a better classification was obtained with fuzzy systems in both type-1 and IT2FS with Gaussian functions, with the IT2FS model showing the best results. When the BSA algorithm was compared with the CSA algorithm, better results were obtained with the former algorithm.

With the experimentation carried out, based on the obtained results, a reliable fuzzy classifier can be provided, which correctly classifies the different heart rate levels that a patient can have based on their age and heart rate tendency provided by a modular neuronal network, and which, when used by an expert, will provide an accurate final diagnosis of any condition that the patient may have.

When comparing the proposed fuzzy system with some of those found in the literature, we could observe that the granulation in the membership functions is carried out in a simpler way, giving our fuzzy system a more extensive pulse level classification.

As future work, we envision the following: general type-2 fuzzy logic can be used to enhance uncertainty management in the fuzzy model of diagnosis, other optimization methods can be used for comparison of results, and the model of diagnosis can be adapted to other medical diagnostic problems.

Author Contributions: Investigation, and developed the model I.M. and P.M.; Methodology and validate J.C.G and G.P.A.

Funding: This research was funded by Consejo Nacional de Ciencia y Tecnología [246774].

Acknowledgments: We would like to express our gratitude to the Consejo Nacional de Ciencia y Tecnologia and Tecnologico Nacional de Mexico/Tijuana Institute of Technology for the facilities and resources granted for the development of this research.

Conflicts of Interest: The authors declare no conflicts of interest.

\section{References}

1. Carvajal, O.R.; Castillo, O.; Soria, J. Optimization of Membership Function Parameters for Fuzzy Controllers of an Autonomous Mobile Robot Using the Flower Pollination Algorithm. J. Autom. Mob. Robot. Intell. Syst. 2018, 12, 44-49.

2. Karami, Y.; Fathy, M.; Khakzad, H.; Shirazi, H.; Arab, S. Protein structure prediction using bio-inspired algorithm: A review. In Proceedings of the 16th CSI International Symposium on Artificial Intelligence and Signal Processing (AISP 2012), Shiraz, Iran, 2-3 May 2012; pp. 201-206.

3. Sari, I.R.F. Bioinspired algorithms for Internet of Things network. In Proceedings of the 20174 th International Conference on Information Technology, Computer, and Electrical Engineering (ICITACEE), Semarang, Indonesia, 18-19 October 2017; p. 1.

4. Domanal, S.; Guddeti, R.M.; Buyya, R. A Hybrid Bio-Inspired Algorithm for Scheduling and Resource Management in Cloud Environment. IEEE Trans. Serv. Comput. 2017, 10, 1-14. [CrossRef]

5. Lagunes, M.L.; Castillo, O.; Soria, J. Methodology for the Optimization of a Fuzzy Controller Using a Bio-inspired Algorithm. In Fuzzy Logic in Intelligent System Design; Springer: Cham, Switzerland, 2018; pp. 131-137.

6. Deng, Y.; Liu, Y.; Zhou, D. An Improved Genetic Algorithm with Initial Population Strategy for Symmetric TSP. Math. Probl. Eng. 2015, 2015, 212794. [CrossRef] 
7. Couceiro, M.; Ghamisi, P. Particle Swarm Optimization. In Fractional Order Darwinian Particle Swarm Optimization: Applications and Evaluation of an Evolutionary Algorithm; Couceiro, M., Ghamisi, P., Eds.; Springer International Publishing: Cham, Switzerland, 2016; pp. 1-10.

8. Yang, X.S.; Karamanoglu, M.; He, X. Flower pollination algorithm: A novel approach for multiobjective optimization. Eng. Optim. 2014, 46, 1222-1237. [CrossRef]

9. Yu, J.J.Q.; Li, V.O.K. A social spider algorithm for global optimization. Appl. Soft Comput. 2015, 30, $614-627$. [CrossRef]

10. Meng, X.-B.; Gao, X.Z.; Lu, L.; Liu, Y.; Zhang, H. A new bio-inspired optimisation algorithm: Bird Swarm Algorithm. J. Exp. Theor. Artif. Intell. 2016, 28, 673-687. [CrossRef]

11. Ahmad, M.; Javaid, N.; Niaz, I.A.; Shafiq, S.; Rehman, O.U.; Hussain, H.M. Application of Bird Swarm Algorithm for Solution of Optimal Power Flow Problems. In Complex, Intelligent, and Software Intensive Systems; Springer: Cham, Switzerland, 2019; pp. 280-291.

12. Cai, L.; Zhang, Y.; Ji, W. Variable Strength Combinatorial Test Data Generation Using Enhanced Bird Swarm Algorithm. In Proceedings of the 2018 19th IEEE/ACIS International Conference on Software Engineering, Artificial Intelligence, Networking and Parallel/Distributed Computing (SNPD), Busan, Korea, 27-29 June 2018; pp. 391-398.

13. Ismail, F.H.; Houssein, E.H.; Hassanien, A.E. Chaotic Bird Swarm Optimization Algorithm. In Proceedings of the International Conference on Advanced Intelligent Systems and Informatics 2018, Cairo, Egypt, 1-3 September 2018; pp. 294-303.

14. Mohamed, K.A.; Hussein, E.M. Malaria Parasite Diagnosis using Fuzzy Logic. Int. J. Sci. Res. 2016, 5, 807-809.

15. Asl, A.A.S.; Zarandi, M.H.F. A Type-2 Fuzzy Expert System for Diagnosis of Leukemia. In Fuzzy Logic in Intelligent System Design; Springer: Cham, Switzerland, 2018; pp. 52-60.

16. Sotudian, S.; Zarandi, M.H.F.; Turksen, I.B. From Type-I to Type-II Fuzzy System Modeling for Diagnosis of Hepatitis. Int. J. Comput. Inf. Eng. 2016, 10, 1280-1288.

17. Zarandi, M.H.F.; Khadangi, A.; Karimi, F.; Turksen, I.B. A Computer-Aided Type-II Fuzzy Image Processing for Diagnosis of Meniscus Tear. J. Digit. Imaging 2016, 29, 677-695. [CrossRef]

18. American Heart Association. 2015. Available online: http://www.heart.org/HEARTORG/Conditions/ HighBloodPressure/High-Blood-Pressure-or-Hypertension_UCM_002020_SubHomePage.jsp (accessed on 15 October 2018).

19. Marchione, V. Healthy Resting Heart Rate by Age for Men and Women. Bel Marra Health. 7 January 2018. Available online: https:/ / www.belmarrahealth.com/resting-heart-rate-chart-factors-influence-heart-rateelderly/ (accessed on 12 August 2018).

20. Ioannou, P.; Velegraki, M.; Soundoulounaki, S.; Gikas, A.; Kofteridis, D.P. An Unexpected Cause of Bradycardia in a Patient with Bacterial Meningitis. Case Rep. Med. 2017, 2017, 4297372. [CrossRef] [PubMed]

21. Gopinathannair, R.; Olshansky, B. Management of tachycardia. F1000Prime Rep. 2015, 7, 60. [CrossRef]

22. Rosendorff, C. Essential Cardiology: Principles and Practice; Springer: New York, NY, USA, 2013.

23. Texas Heart Institute. High Blood Pressure (Hypertension). 2017. Available online: https://www.texasheart. org/heart-health/heart-information-center/topics/high-blood-pressure-hypertension/ (accessed on 8 October 2018).

24. Mancia, G.; Grassi, G.; Redon, J. Manual of Hypertension of the European Society of Hypertension; CRC Press: Boca Raton, FL, USA, 2014.

25. Giuseppe, M.; Guido, G. The Autonomic Nervous System and Hypertension. Circ. Res. 2014, 114, $1804-1814$.

26. Mancia, G.; Fagard, R.; Narkiewicz, K.; Redon, J.; Zanchetti, A.; Böhm, M.; Christiaens, T.; Cifkova, R.; de Backer, G.; Dominiczak, A.; et al. 2013 ESH/ESC guidelines for the management of arterial hypertension: The Task Force for the management of arterial hypertension of the European Society of Hypertension (ESH) and of the European Society of Cardiology (ESC). Eur. Heart J. 2013, 34, 2159-2219.

27. Zadeh, L.A. Fuzzy sets. Inf. Control 1965, 8, 338-353. [CrossRef]

28. Melin, P. Modular Neural Networks and Type-2 Fuzzy Systems for Pattern Recognition; Springer: Berlin/Heidelberg, Germany, 2012.

29. Duodu, Q.; Panford, J.K.; Hafron-acquah, J.B. Designing Algorithm for Malaria Diagnosis using Fuzzy Logic for Treatment (AMDFLT) in Ghana. Int. J. Comput. Appl. 2014, 91, 22-27. [CrossRef] 
30. Morsi, I.; el Gawad, Y.Z.A. Fuzzy logic in heart rate and blood pressure measuring system. In Proceedings of the IEEE Sensors Applications Symposium Proceedings, Galveston, TX, USA, 19-21 February 2013; pp. 113-117.

31. Nohria, R.; Mann, P.S. Diagnosis of Hypertension using Adaptive Neuro-Fuzzy Inference System. Int. J. Comput. Sci. Technol. 2015, 8491, 36-40.

32. Sikchi, S.; Sikchi, S.; Ali, M. Design of fuzzy expert system for diagnosis of cardiac diseases. Int. J. Med. Sci. Public Heal. 2013, 2, 56-61. [CrossRef]

33. Udo, E.U.; Oparaaku, O.U. Fuzzy Logic System for Fetal Heart Rate Determination. Int. J. Eng. Res. 2015, 4, 60-63.

34. Pabbi, V. Fuzzy Expert System for Medical Diagnosis. Int. J. Sci. Res. Publ. 2015, 5, 1-7.

35. Miramontes, I.; Martínez, G.; Melin, P.; Prado-Arechiga, G. A Hybrid Intelligent System Model for Hypertension Risk Diagnosis. In Fuzzy Logic in Intelligent System Design; Springer: Cham, Switzerland, 2018; pp. 202-213.

36. Melin, P.; Miramontes, I.; Prado-Arechiga, G. A hybrid model based on modular neural networks and fuzzy systems for classification of blood pressure and hypertension risk diagnosis. Expert Syst. Appl. 2018, 107, 146-164. [CrossRef]

37. Miramontes, I.; Martínez, G.; Melin, P.; Prado-Arechiga, G. A Hybrid Intelligent System Model for Hypertension Diagnosis. In Nature-Inspired Design of Hybrid Intelligent Systems; Melin, P., Castillo, O., Kacprzyk, J., Eds.; Springer International Publishing: Cham, Switzerland, 2017; pp. 541-550.

38. Guzman, J.C.; Melin, P.; Prado-Arechiga, G. Design of an optimized fuzzy classifier for the diagnosis of blood pressure with a new computational method for expert rule optimization. Algorithms 2017, 10, 79. [CrossRef]

39. Guzmán, J.C.; Melin, P.; Prado-Arechiga, G. Neuro-Fuzzy Hybrid Model for the Diagnosis of Blood Pressure. In Nature-Inspired Design of Hybrid Intelligent Systems; Melin, P., Castillo, O., Kacprzyk, J., Eds.; Springer International Publishing: Cham, Switzerland, 2017; pp. 573-582.

40. Guzmán, J.C.; Melin, P.; Prado-Arechiga, G. Design of a Fuzzy System for Diagnosis of Hypertension. In Design of Intelligent Systems Based on Fuzzy Logic, Neural Networks and Nature-Inspired Optimization; Springer International Publishing: Cham, Switzerland, 2015; pp. 517-526.

41. Melin, P.; Prado-Arechiga, G.; Miramontes, I.; Guzman, J.C. Classification of nocturnal blood pressure profile using fuzzy systems. J. Hypertens. 2018, 36, e111-e112. [CrossRef]

42. O'Brien, E.; Parati, G.; Stergiou, G. Ambulatory Blood Pressure Measurement. Hypertension 2013, 62, 988-994. [CrossRef]

43. Mart, D. Optimal tuning of a networked linear controller using a multi-objective Genetic Algorithm and its application to one complex electromechanical. Int. J. Innov. Comput. Inf. Control 2009, 5, 3405-3414.

44. Saadat, J.; Moallem, P.; Koofigar, H. Training echo estate neural network using harmony search algorithm. Int. J. Artif. Intell. 2017, 15, 163-179.

45. Precup, R.; David, R.; Petriu, E.M. Grey Wolf Optimizer Algorithm-Based Tuning of Fuzzy Control Systems with Reduced Parametric Sensitivity. IEEE Trans. Ind. Electron. 2017, 64, 527-534. [CrossRef]

46. Vrkalovic, S.; Lunca, E.; Borlea, I. Model-Free Sliding Mode and Fuzzy Controllers for Reverse Osmosis Desalination Plants. Int. J. Artif. Intell. 2018, 16, 208-222.

(C) 2018 by the authors. Licensee MDPI, Basel, Switzerland. This article is an open access article distributed under the terms and conditions of the Creative Commons Attribution (CC BY) license (http://creativecommons.org/licenses/by/4.0/). 